\title{
Sensory Axon Growth Requires Spatiotemporal Integration of CaSR and TrkB Signaling
}

\author{
DRonja Markworth, ${ }^{1,2,3}$ Youri Adolfs, ${ }^{4}$ Vivian Dambeck, ${ }^{1,3}$ @Lars M. Steinbeck, ${ }^{3}{ }^{\circledR}$ Muriel Lizé, ${ }^{1}$ \\ (D) R. Jeroen Pasterkamp, ${ }^{4}$ Mathias Bähr, ${ }^{1}$ Camin Dean, ${ }^{2}$ and Catja Burk ${ }^{1,2,3}$ \\ ${ }^{1}$ Department of Neurology, University Medical Center Göttingen, 37075 Göttingen, Germany, ${ }^{2}$ European Neuroscience Institute, 37077 Göttingen, \\ Germany, ${ }^{3}$ Center for Biostructural Imaging of Neurodegeneration, 37075 Göttingen, Germany, and ${ }^{4}$ Department of Translational Neuroscience, University \\ Medical Center Utrecht, Utrecht University, 3584 CG Utrecht, The Netherlands
}

Neural circuit development involves the coordinated growth and guidance of axons. During this process, axons encounter many different cues, but how these cues are integrated and translated into growth is poorly understood. In this study, we report that receptor signaling does not follow a linear path but changes dependent on developmental stage and coreceptors involved. Using developing chicken embryos of both sexes, our data show that calcium-sensing receptor (CaSR), a G-protein-coupled receptor important for regulating calcium homeostasis, regulates neurite growth in two distinct ways. First, when signaling in isolation, CaSR promotes growth through the PI3-kinase-Akt pathway. At later developmental stages, CaSR enhances tropomyosin receptor kinase B (TrkB)/BDNF-mediated neurite growth. This enhancement is facilitated through a switch in the signaling cascade downstream of CaSR (i.e., from the PI3-kinase-Akt pathway to activation of GSK3 $\alpha$ Tyr279). TrkB and CaSR colocalize within late endosomes, cotraffic and coactivate GSK3, which serves as a shared signaling node for both receptors. Our study provides evidence that two unrelated receptors can integrate their individual signaling cascades toward a nonadditive effect and thus control neurite growth during development.

Key words: CaSR; cosignaling; GSK3; neurite growth; signal integration; TrkB

\section{Significance Statement}

This work highlights the effect of receptor coactivation and signal integration in a developmental setting. During embryonic development, neurites grow toward their targets guided by cues in the extracellular environment. These cues are sensed by receptors at the surface that trigger intracellular signaling events modulating the cytoskeleton. Emerging evidence suggests that the effects of guidance cues are diversified, therefore expanding the number of responses. Here, we show that two unrelated receptors can change the downstream signaling cascade and regulate neuronal growth through a shared signaling node. In addition to unraveling a novel signaling pathway in neurite growth, this research stresses the importance of receptor coactivation and signal integration during development of the nervous system.

\section{Introduction}

The development of the nervous system requires axons and growth cones to extend through a complex environment. This

Received Jan. 4, 2019; revised May 12, 2019; accepted May 15, 2019.

Author contributions: R.M., Y.A., V.D., M.L., and K.B. performed research; R.M., L.M.S., and K.B. analyzed data; R.M., M.L., R.J.P., M.B., C.D., and K.B. edited the paper; Y.A., R.J.P., and K.B. designed research; K.B. wrote the first draft of the paper; K.B. wrote the paper.

This work was supported by a Dorothea Schloezer fellowship to K.B., an International Max Planck Research School for Neurosciences stipend to R.M., the Center for Nanoscale Microscopy and Molecular Physiology of the Brain to K.B., R.M., M.B., and C.D., and The Netherlands Organization for Scientific Research to R.J.P. We thank Michael Levine for providing the GFP-CaSR full-length and GFP-R1850 constructs; Moses Chao for the TrkB-RFP construct; and Luisa Leiss for helping with image acquisition.

The authors declare no competing financial interests.

Correspondence should be addressed to Katja Burk at kburk@gwdg.de.

https://doi.org/10.1523/JNEUROSCI.0027-19.2019

Copyright $\odot 2019$ the authors direction-mediated process allows growth cones to find and innervate their appropriate targets and form functional synapses.

Axon pathfinding demands neurons to respond to long- and short-range signals, either repulsive or attractive (Dickson, 2002; Wen and Zheng, 2006; Bashaw and Klein, 2010) in addition to factors accelerating or inhibiting axonal extension (Filbin, 2006). Remarkably, our nervous system contains trillions of connections but only about a hundred of growth/guidance cues. Therefore, one important question is how this relatively small number of cues coordinates the wiring of a disproportionally large number of connections. Evidence is emerging that the axon growth/ guidance cues are diversified and spatiotemporally controlled, expanding the number of guidance decisions they can mediate.

The signals involved for fine-tuning neuronal growth are diversified (e.g., through several ligands binding to one receptor) 
(Chao, 2003; Zhou et al., 2008; Dudanova and Klein, 2013) or ligands binding directly to their receptors or through a coreceptor (Chauvet et al., 2007; Bellon et al., 2010; Song et al., 2015; Mire et al., 2018), eventually leading to differential changes of the cytoskeleton (Bashaw and Klein, 2010).

Additionally, spatial and temporal expression of receptors and coreceptors modulates responses depending on developmental states (Dickson and Gilestro, 2006; Mire et al., 2012, 2018). Furthermore, several studies have reported that signaling cascades interact to generate complex cellular behaviors (Bourne and Nicoll, 1993; Cornell and Kimelman, 1994; Prehoda and Lim, 2002; McClean et al., 2007). These interactions of downstream signaling cascades are classified into two categories: additive and nonadditive. Additive signaling is integrated as a sum of positive and/or negative signals. Nonadditive effects are quantitatively or qualitatively different from the sum of signals (Dudanova and Klein, 2013). These nonadditive effects can include synergistic, hierarchical, and permissive subclasses of interactions (see Morales and Kania, 2017). However, despite recent progress, our understanding of how signal integration induces growth and guidance remains incomplete.

To address these questions, we used the calcium-sensing receptor (CaSR), a G-protein-coupled receptor, monitoring extracellular free ionized calcium in organs involved in calcium homeostasis. Recently, unexpected functions of CaSR in the nervous system were found (Vizard et al., 2008; Ruat and Traiffort, 2013; Jones and Smith, 2016).

Vizard et al. (2008) reported that CaSR enhances neurotrophic-mediated growth of neurons from the mouse superior cervical ganglion (SCG). The authors used neurons of the SCG, which require NGF/TrkA signaling for growth and survival. When CaSR was activated additionally to TrkA, neurite length increased compared with TrkA activation alone.

To answer how CaSR increases neurotrophin-facilitated growth, we used the chicken nodose ganglia as a model system. During early chick development, nodose neurons do not require neurotrophins for growth and survival (Davies, 1989) but rather become dependent on BDNF-TrkB signaling upon target innervation (Vogel and Davies, 1991; Robinson et al., 1996). Early nodose neurons respond to BDNF after $\sim 72 \mathrm{~h}$ in culture, which was suggested to be the time when neurons innervate their targets in vivo (Vogel and Davies, 1991; Robinson et al., 1996). We used this developmental switch to study CaSR signaling in the absence and presence of BDNF-TrkB signaling.

Our data show that, when CaSR signals in isolation, it routes into Rab7-positive endosomes and regulates axonal growth through activation of Akt. Later in development with expression and coactivation of TrkB, CaSR colocalizes with TrkB in Rab7positive endosomes, cotraffics and coimmunoprecipitates with TrkB, and enhances BDNF-mediated growth through shared activation on GSK3.

In summary, this study reports several novel observations. First, CaSR regulates BDNF-independent neurite growth. Second, downstream targets shift, depending on developmental stages. Further, we show how two unrelated receptors integrate their signaling in a nonadditive manner on a shared signaling-node, namely, GSK3 $\alpha$ tyrosine (Tyr) 279 and GSK3 $\beta$ serine (Ser) 9. Last, both receptors act on the activity state of GSK3. This activity state, in turn, affects the phosphorylation state of Tau, which modulates microtubule assembly and disassembly.

\section{Materials and Methods}

All research involving animals was approved by, and done in accordance with, the Institutional Animal Care and Ethics Committees of Göttingen University and with German animal welfare laws, and in accordance with the Animals Scientific Procedures Act of 1986 (UK). To generate mouse embryonic fibroblasts (MEFs), embryos were isolated from gestating C57BL/6N females at embryonic day E13.5 after fertilization by male C57BL/6N, minced, and taken into culture in DMEM.

Calculation of calcium concentration. DMEM (catalog \#31966-021, Invitrogen) was used to culture chicken nodose neurons, which has a standard calcium concentration of $1.8 \mathrm{~mm}$ (details on calcium concentrations for DMEM can be found on the manufacturer's website). To increase calcium levels by $0.5 \mathrm{~mm}(1.8-2.3 \mathrm{~mm})$, the amount of $1 \mathrm{M} \mathrm{CaCl}$ needed for the respective volume was calculated and added to the media. To decrease calcium levels by $1.1 \mathrm{~mm}(1.8-0.7 \mathrm{~mm})$, the amount of $0.5 \mathrm{M}$ EGTA needed for the respective volume used was calculated and added to the media.

Culture of chicken nodose neurons. Nodose ganglia were removed from 10-12 chicken embryos of both sexes per dissection (for immunostainings and Western blots) and transferred to ice-cold HBSS buffer and kept on ice during dissection. Nodose ganglia were then transferred into $0.25 \%$ trypsin solution and incubated for $10 \mathrm{~min}$ at $37^{\circ} \mathrm{C}$. During the incubation, $1 \mathrm{ml}$ "plating medium" (DMEM-containing glucose, pyruvate, glutamine, penicillin/streptomycin, and 10\% FBS) was filled into each well of 24-well plates containing poly-L-ornithine and laminincoated coverslips. After incubation, enzyme solution was removed and 5 $\mathrm{ml}$ plating medium added and incubated for $5 \mathrm{~min}$ at $37^{\circ} \mathrm{C}$ for neutralization. Plating medium was removed, and nodose ganglia were washed twice with plating medium; $2 \mathrm{ml}$ plating medium was added to the dissected ganglia, and the tissue was dissociated with a $1 \mathrm{ml}$ pipette; $400 \mu \mathrm{l}$ of cell suspension was added to each well. Neurons were then kept at $37^{\circ} \mathrm{C}$, $5 \% \mathrm{CO}_{2}$ and $100 \%$ humidity in calcium concentrations specified for each experiment below.

Survival assay. Nodose neurons were dissected as described above at different developmental time points and kept in "plating medium" in 35 mm tissue culture dishes (Greiner Cellstar, catalog \#M9312-100EA) coated with poly-L-ornithine and laminin at a density of $\sim 2000$ neurons per dish. After an initial count, which was performed $2 \mathrm{~h}$ after plating for neurons to settle on the bottom of the culture dish, half of the dishes were treated with $10 \mathrm{ng} / \mathrm{ml} \mathrm{BDNF}$ and half of the dishes served as controls. The number of neurons within a predefined $12 \times 12 \mathrm{~mm}$ area in the middle of the culture dish were counted again $24 \mathrm{~h}$ after plating. The number of neurons surviving at these times was expressed as a percentage of the initial number of neurons. Three to five independent cultures were analyzed for all conditions, and the data shown were compiled from separate experiments.

Measuring neurite length. Nodose neurons were dissected as described above and plated on acidified glass coverslips coated with poly-Lornithine and laminin at a density of $\sim 5000$ neurons per well and placed in 24-well tissue culture dishes (Greiner Cellstar, catalog \#M9312100EA). Neurons were grown at $5 \% \mathrm{CO}_{2}$ and $37^{\circ} \mathrm{C}$ for $24 \mathrm{~h}$ in "plating medium" adjusted to the respective calcium concentrations $0.7,1.1,1.3$, or $2.3 \mathrm{~mm}$ calcium, $0.7 \mathrm{~mm}+10 \mu \mathrm{M}$ CaSR agonist (R568 hydrochloride), or $2.3 \mathrm{~mm}+10 \mu \mathrm{M}$ CaSR antagonist (Calhex 231 hydrochloride), respectively (for Stage 20 nodose neurons). Stage 30 nodose neurons were cultured in $10 \mathrm{ng} / \mathrm{ml}$ BDNF (R\&D Systems) and the following conditions: $0.7 \mathrm{~mm}, 0.7 \mathrm{~mm}+$ CaSR agonist (R568 hydrochloride), $2.3 \mathrm{~mm}$ or $2.3 \mathrm{~mm}+$ CaSR antagonist (Calhex 231 hydrochloride). Inhibitors for growth pathways were used as follows: $50 \mu \mathrm{M}$ PI3-kinase inhibitor LY294002 (Sigma-Aldrich, catalog \#L9908), $10 \mu \mathrm{M}$ GSK3 inhibitor BIO (Sigma-Aldrich, catalog \#B1686), and $10 \mu \mathrm{M}$ MEK1/2 inhibitor PD98059 (Sigma-Aldrich catalog \#P215). Caspase inhibitor was used at $25 \mu \mathrm{M}$ (caspase inhibitor III/Boc-D-FMK, Merck Millipore, catalog \#218745).

After $24 \mathrm{~h}$, neurons were fixed in $4 \%$ PFA, washed $3 \times$ in PBS, and stained with tubulin antibody as described in Immunocytochemistry or directly stained with CalceinAM (Thermo Fisher Scientific, catalog \#C3100MP). 
Neurite length was measured using the Fiji PlugIn Simple Neurite Tracer (RRID:SCR_016566). In early, bipolar neurons, the entire length of neurons was measured, whereas in late development we took the sum of lengths from all neurites of a neuron to stay consistent with our measuring method.

The number of branch points was measured using the Fiji PlugIn Cell Counter (RRID:SCR_002285), which was used to manually mark each branch point on a neuron.

For in ovo measurements, eggs were opened at Stage 18, and a $10 \mu \mathrm{l}$ droplet of agonist/antagonist solution $(100 \mu \mathrm{g} / \mu \mathrm{l})$ or DMSO was added to the region of the nodose and the embryos were incubated for $24 \mathrm{~h}$.

Whole-mount neurofilament staining was performed as follows: Embryos were fixed in 4\% PFA for $2 \mathrm{~h}$. Subsequently, embryos were washed 3 times (30 min each) in PBS containing 1\% Triton-X and then blocked for $2 \mathrm{~h}$ in PBS containing 1\% Triton-X, 10\% FCS. Then, embryos were blocked overnight in PBS containing 1\% Triton-X, 10\% FCS, and $0.1 \%$ $\mathrm{H}_{2} \mathrm{O}_{2}$. The next day, embryos were washed 3 times in blocking solution (PBS containing 1\% Triton-X, 10\% FCS) and then incubated for $2 \mathrm{~d}$ in antineurofilament antibody (1:50, catalog \#N4142, Sigma-Aldrich, RRID:AB_477272). Following this, embryos were washed 3 times for 30 min each in blocking solution and then incubated for $2 \mathrm{~d}$ in secondary antibody (1:250 catalog \#ab6721, Abcam, RRID:AB_955447). After incubation, embryos were washed 3 times in PBS containing 1\% Triton-X and then incubated in DAB substrate containing $\mathrm{H}_{2} \mathrm{O}_{2}\left(5 \mu \mathrm{H}_{2} \mathrm{O}_{2}\right.$ per 1 $\mathrm{ml}$ of $\mathrm{DAB}$ ) until neurons were clearly visible. DAB solution was removed; embryos were washed in PBS and imaged using a light microscope. The length of nodose neurons was measured from the hindbrain to the tip of the longest neurite (using the Fiji PlugIn Simple Neurite Tracer, RRID:SCR_016566) and normalized to the circumference of the midbrain.

Transfection with Lipofectamine 2000 reagent (for neurite length measurements, TIRF microscopy, and construct expression in N1E-115 cells). RFP-tagged TrkB was obtained from Moses Chao; CaSR-WT and CaSR-DN R185Q were obtained from Michel Levine. For expression in chicken neurons, pCAG-GFP vector (Addgene, 11150) was cut with Smal and NotI, which removed GFP from the pCAG-GFP vector, which was replaced by CaSR-eGFP (restricted from a CaSR-eGFP-N1 vector) or the R185Q-GFP via ligation. Hereby, CaSR was expressed under a $\beta$-actin promoter.

For nodose neurons, neurons were dissected at Stage 20 and kept in "plating medium" for $2 \mathrm{~h}$ to settle before being transfected on the day of dissection. Because neurons become dependent on BDNF when kept in culture after Stage 24, we allowed neurons to grow for $30 \mathrm{~h}$ in plating medium at $37^{\circ} \mathrm{C} 5 \% \mathrm{CO}_{2}$ for the constructs to be expressed. Because of the short expression time, we enhanced the GFP signal after fixation using an anti-GFP antibody (ChromoTek, catalog \#3h9-100, RRID: AB_10773374; details in Immunocytochemistry).

MEFs and N1E-115 cells were transfected at 70\% density, and expression was allowed for 2-3 d.

Lipofectamine transfection was performed according to the manufacturer's instructions. For Solution A, $1 \mu$ l of Lipofectamine 2000 (Thermo Fisher Scientific) was added to $100 \mu$ l DMEM (Invitrogen) (to be adjusted to the final volume per well, depending on the experiment) and incubated for $5 \mathrm{~min}$ at room temperature. For Solution B, $1 \mu \mathrm{g}$ of the desired plasmid was added to $100 \mu \mathrm{l}$ of DMEM (to be adjusted to final volume per well). Then both solutions were mixed and incubated for 20 min at room temperature. In the meantime, "plating medium" from each well was replaced by prewarmed DMEM and stored at $37^{\circ} \mathrm{C}$. After the incubation time, the transfection mixture was added to each well and incubated for $75 \mathrm{~min}$ at $37^{\circ} \mathrm{C}$. Finally, the transfection mixture was removed, and the stored "plating medium" was added back to the cultures.

TIRF microscopy. Transfected MEFs were trypsinized and replated on a MatTek $35 \mathrm{~mm}$ glass-bottom poly-D-lysine-coated tissue culture dish. On the day of imaging, MEFs were kept in "starving medium" (DMEM without penicillin/streptomycin and FBS), which was adjusted to $0.7 \mathrm{~mm}$ calcium for $20 \mathrm{~min}$. Then, "starving media" was refreshed serving as a control, and MEFs were placed on an AxioObserver Z1 TIRF microscope (Carl Zeiss) with an Evolve CCD camera (Photometrics) using the $100 \times$ objective and imaged ( $5 \mathrm{~min}$ time-lapse recordings with pictures taken in
$10 \mathrm{~s}$ intervals). Subsequently, after imaging control conditions, MEFs were stimulated with $10 \mu \mathrm{m}$ of CaSR agonist R568 hydrochloride (Tocris Bioscience), $10 \mathrm{ng} / \mathrm{ml}$ BDNF (R\&D Systems), or a combination of both, and imaged during 5 min time-lapse recordings with pictures taken in $10 \mathrm{~s}$ intervals. From time-lapse recordings, we analyzed colocalization and generated kymographs. Colocalization was evaluated using the Fiji colocalization plugin measuring the Pearson correlation coefficient (Fiji, RRID:SCR_002285). Kymographs were generated using the Fiji PlugIn KymographBuilder (RRID for KymographBuilder PlugIn not available, for Fiji software: RRID:SCR_002285). Comovement was evaluated by quantifying the percentage of moving tracks, where moving was defined as a deflection $>20$ degrees from vertical for at least $30 \mathrm{~s}$.

Colocalization of CaSR and TrkB with endosomes (for immunocytochemistry). Nodose neurons were dissected as described above and plated on acidified glass coverslips coated with poly-L-ornithine and laminin at a density of $\sim 5000$ neurons per well and placed in 24-well tissue culture dishes (Greiner Cellstar, catalog \#M9312-100EA). Neurons were grown at $5 \% \mathrm{CO}_{2}$ and $37^{\circ} \mathrm{C}$ for $24 \mathrm{~h}$ in "plating medium" ( $1.8 \mathrm{~mm}$ calcium) and, for Stage 30 nodose neurons, in the presence of $10 \mathrm{ng} / \mathrm{ml} \mathrm{BDNF}$ (R\&D Systems). After $24 \mathrm{~h}$, neurons were starved for $20 \mathrm{~min}$ using "starving medium" adjusted to $0.7 \mathrm{~mm}$ calcium. Subsequently, neurons were stimulated for 20 min with $10 \mu \mathrm{M}$ of CaSR agonist R568 hydrochloride or 10 $\mathrm{ng} / \mathrm{ml}$ BDNF. Nonstimulated neurons in "starving medium" served as a control. Following this, neurons were fixed in 4\% PFA, washed 3 times in PBS, and stained with antibodies as described in Immunocytochemistry.

Immunocytochemistry. Nodose neurons were dissected as described above and fixed on DIV2 with $4 \%$ PFA. Cells were blocked in $0.1 \%$ Triton and 2\% FBS for at least $60 \mathrm{~min}$ on a shaker at room temperature. Cells were then incubated in primary antibody $(1 / 1000)$ overnight in blocking solution at $4^{\circ} \mathrm{C}$. Antibodies used include the following: anti-GFP (rat) 3h9-100 ChromoTek (RRID:AB_10773374), EEAl (rabbit), catalog \#PA5-17228 Thermo Fisher Scientific (RRID:AB_11004515); Rab5 (mouse) catalog \#108001 Synaptic Systems (RRID:AB_2619777); Rab11 (rabbit) catalog \#ab3612 Abcam (RRID:AB_10861613); Rab7 (rabbit) catalog \#D95F2 Cell Signaling Technology (RRID:AB_1904103); Lamp1 (rabbit) catalog \#9091 Cell Signaling Technology (RRID:AB_2687579); TrkB (chicken) catalog \#G1561 Promega (RRID:AB_430846), CaSR (goat) catalog \#F19 Santa Cruz Biotechnology (RRID:AB_2290992), and $\beta$ III-tubulin (mouse) catalog \#ab78078 Abcam (RRID:AB_2256751). Secondary antibodies were AlexaFluor-488 goat anti-mouse catalog \#A11001 Thermo Fisher Scientific (RRID:AB_2534069); AlexaFluor-488 goat anti-rabbit catalog \#A-11008, Thermo Fisher Scientific (RRID: AB_143165); AlexaFluor-546 goat anti-mouse catalog \#A-11003, Invitrogen (RRID:AB_141370); AlexaFluor-546 goat anti-rabbit catalog \#A11035 Thermo Fisher Scientific (RRID:AB_2534093); AlexaFluor-488 goat anti-chicken catalog \#A-11039 Invitrogen (RRID:AB_142924); AlexaFluor-488 donkey anti-goat catalog \#A-11055 Thermo Fischer Scientific (RRID:AB_2534102); and AlexaFluor-546 donkey anti-rabbit catalog \#A10040 Thermo Fischer Scientific (RRID:AB_2534016).

After three washes of $\sim 5$ min with PBS, secondary antibodies were applied for $120 \mathrm{~min}$ at room temperature. Cells were washed three times for 10 min with PBS followed by a quick rinse in $\mathrm{ddH}_{2} \mathrm{O}$, and then the coverslips were immediately mounted using Mowiol 4-88. Images were acquired with the LSM710 confocal microscope (Carl Zeiss) using the $63 \times$ or $100 \times$ objective, including $Z$ stacks. Gain was set so that there was no saturation in the images. On average, 3 optical slices were taken per image at a $1 \mu \mathrm{m}$ interval between slices. Average optical thickness was $0.5 \mu \mathrm{m}$

Colocalization was analyzed in single optical slices with the Fiji colocalization plugin measuring the Pearson correlation coefficient (Fiji, RRID:SCR_002285).

Transfection with calcium phosphate (for coimmunoprecipitation). HEK293 cells were transfected using a calcium phosphate protocol to receive high levels of transfection for biochemistry experiments. Transfection buffer containing $274 \mathrm{~mm} \mathrm{NaCl}, 10 \mathrm{~mm} \mathrm{KCl}, 1.4 \mathrm{mM} \mathrm{Na}_{2} \mathrm{HPO}_{4}$, $15 \mathrm{~mm}$ glucose, and $42 \mathrm{~mm}$ HEPES was added dropwise to a solution of DNA $(10 \mu \mathrm{g} / 10 \mathrm{~cm}$ dish $)$ and $250 \mathrm{~mm} \mathrm{CaCl}_{2}$. The resulting mix was kept in the dark for $20 \mathrm{~min}$ and then added onto HEK cells and left on for 1-2 $\mathrm{d}$ to allow expression. 
Immunoprecipitation. HEK and N1E-115 cells (plated in $10 \mathrm{~cm}$ culture dishes in DMEM containing 10\% FBS and 1\% penicillin/streptomycin) were transfected with TrkB-RFP and CaSR-GFP constructs as described above. Two days after transfection, cells were washed with ice-cold PBS and subsequently lysed using $1 \mathrm{ml}$ of "lysis buffer" containing $10 \mathrm{mM}$ Tris/HCl, pH 7.5, $150 \mathrm{~mm} \mathrm{NaCl,} 0.5$ mм EDTA, and 0.5\% NP-40. Protease inhibitors (Sigma-Aldrich, catalog \#P8340) were added just before application. RFP-Trap, GFP-Trap (ChromoTek), or control beads (coated with rabbit IgG, Sigma-Aldrich, catalog \#A8914) were washed 3 times with "wash buffer" ("lysis buffer" without NP-40) before use; $60 \mu \mathrm{l}$ was taken from the lysate for the input control and mixed with $60 \mu \mathrm{l}$ of $2 \times$ sample buffer. The whole leftover lysate was added to the beads for $3 \mathrm{~h}$, followed by removal of the lysate from the beads and recovering $60 \mu \mathrm{l}$ for unbound controls, mixed with $60 \mu$ of $2 \times$ sample buffer. Beads were then washed 3 times with "wash buffer." Following washing, $100 \mu \mathrm{l}$ of sample buffer was added to the beads. All samples were boiled for $10 \mathrm{~min}$ and then loaded on a $10 \%$ SDS-PAGE gel.

Western blots. Nodose ganglia were dissected as described above and plated in high density in 96-well plates (Greiner Cellstar, catalog \#M0687-100EA, 2 ganglia per 1 well) coated with poly-L-ornithine and laminin. After allowing neurons to grow for $24 \mathrm{~h}$ in "plating medium" (containing $10 \mathrm{ng} / \mathrm{ml}$ BDNF for neurons after Stage 22), neurons were starved in "starving medium" adjusted to $0.7 \mathrm{~mm}$ calcium for $20 \mathrm{~min}$. Following starvation, medium was removed (except for control) and replaced by respective stimulants, which were either $10 \mu \mathrm{M}$ CaSR agonist in $0.7 \mathrm{~mm}$ "starving medium" (R568 hydrochloride, catalog \#3815, Tocris Bioscience), $10 \mu \mathrm{M}$ antagonist in $2.3 \mathrm{~mm}$ "starving medium" (Calhex 231 hydrochloride, catalog \#4387, Tocris Bioscience), $10 \mathrm{ng} / \mathrm{ml}$ BDNF in $0.7 \mathrm{~mm}$ "starving medium" (catalog \#PHC7074, Invitrogen) or $10 \mathrm{ng} / \mathrm{ml}$ BDNF and $10 \mu \mathrm{M}$ CaSR agonist in $0.7 \mathrm{~mm}$ "starving medium" for 5, 15, and $30 \mathrm{~min}$. Subsequently, neurons were lysed using "lysis buffer." Samples were prepared for SDS-PAGE by adding $2 \times$ sample buffer and boiling for $10 \mathrm{~min}$ followed by loading on a $10 \%, 12 \%$, or $15 \%$ SDS-PAGE gel, depending on the target protein size. Gels were transferred and developed using following antibodies: TrkB (rabbit), catalog \#AB9872, Millipore (RRID:AB_2236301); $\beta$-actin, catalog \#251003, Synaptic Systems (RRID:AB_11042458); p-AKT Ser473, catalog \#TA328006, Acris, $\beta$ IIItubulin, catalog \#ab78078, Abcam (RRID:AB_2256751); p-Y279/216GSK3, catalog \#ab68476, Abcam (RRID:AB_10013745); p-S9-GSK3, catalog \#5558, Cell Signaling Technology (RRID:AB_10013750); antiRFP 5f8-100, ChromoTek (RRID:AB_2336064), anti-GFP 3h9-100, ChromoTek (RRID:AB_10773374); p-Tau ab109390, Abcam (RRID: AB_10860822); and $\beta$-catenin, catalog \#4270, Cell Signaling Technology (RRID:AB_1903918). Secondary antibodies were IRDye 800RD donkey anti-mouse, catalog \#925-32210, LI-COR Biosciences (RRID: AB_2687825) and IRDye 800RD donkey anti-rabbit, catalog \#92532211, LI-COR Biosciences (RRID:AB_2651127). Image acquisition was performed using Odyssey Imaging Systems, Odyssey CLx (RRID: SCR_014579), quantification was done using CAPT (RRID: SCR_016305).

Experimental design and statistical analysis. All experiments on chicken nodose neurons were performed using chicken embryos of both sexes either at Stage 22 or Stage 30. For individual experiments, 10-12 embryos were dissected from 3-5 independent experiments as indicated below. Statistical analysis was performed by GraphPad Prism 6.0 software (RRID:SCR_002798); level of significance was set at $p<0.05$. For multiple-comparisons, one-way ANOVAs were performed followed by post hoc Sidak's, Tukey's, or Dunnett's, as appropriate and indicated below and in the figure legends. Since GraphPad Prism 6.0 software does not provide exact $p$ values under a range of $p<0.0001$, we refer to standard $p$ value ranges as indicated in the figure legends. Exact $p$ values (when provided) are indicated in Results. Degrees of freedom for each experiment are indicated in the text and in the figure legends.

We performed three individual experiments for dose-response and four independent experiments for CaSR agonist and antagonist responses at Stage 22 (see Fig. 1D). At Stage 30, we performed five independent experiments for CaSR agonist and antagonist responses (see Fig. $3 D$ ). The number of neurons imaged for these experiments ranged between 25 and 60 neurons per condition and experiment. Neurite length was measured using the Fiji PlugIn Simple Neurite Tracer (RRID: SCR_016566). Significance was determined by one-way ANOVA with post hoc Tukey.

Branch points were counted on images from growth experiments in either 0.7 or $2.3 \mathrm{~mm}$ calcium or the CaSR agonist and antagonist (see Figs. $1 E, 3 F)$. For branch point analysis, we compared two groups by Student's $t$ test and outliers were removed using Tukey's fences.

In vitro transfection was analyzed from three individual experiments. The number of neurons imaged for these experiments ranged between 15 and 30 per condition (see Fig. $1 G$ ). Neurite length was measured using the Fiji PlugIn Simple Neurite Tracer (RRID:SCR_016566). Significance was determined by one-way ANOVA with post hoc Tukey.

In vivo measurements were analyzed from three individual experiments with three or four embryos being used per condition and experiment. Since neurites were on different optical planes, three or four pictures were taken of each embryo. Three or four of the longest neurites were measured per plane and condition and used for analyses (see Fig. 1I). Neurite length was measured using the Fiji PlugIn Simple Neurite Tracer (RRID:SCR_016566). Significance was determined by one-way ANOVA with post hoc Tukey.

For experiments in the presence of PI3-kinase, GSK3, and Mek/Erk inhibitors, we performed four individual experiments (see Figs. $2 B, 4 B$ ). The number of neurons imaged for these experiments ranged between 25 and 60 neurons per condition and experiment. Significance was determined by one-way ANOVA with post hoc Dunnett compared with control (2.3 mm calcium).

Western blots and coimmunoprecipitation experiments were analyzed from 4-6 individual experiments (see Figs. $2 C-E, 4 C-F, 7 A-C$, $9 A-G, 10 A-F)$. Significance was determined by one-way ANOVA with post hoc Tukey (see Figs. 2E, 4E) or Dunnett compared with normalized control ( $0.7 \mathrm{~mm}$ calcium also normalized to each loading control).

Colocalization experiments in neurons were analyzed from three individual experiments (see Figs. $2 F, G, 5 A-D, 6 A-F$ ) by using $15-30$ images (containing two or three optical sections) per condition and experiment. An ROI was identified in a single optical section (excluding nucleus) and measured with the Fiji PlugIn for Pearson's correlation coefficient (Fiji, RRID:SCR_002285). Significance was determined by one-way ANOVA with post hoc Tukey.

Neuronal survival over development was analyzed from three individual experiments per age group. Neurons were counted as described in Materials and Methods. Significance was determined by one-way ANOVA with post hoc Sidak compared with the no factor control of each age group (see Fig. 3C).

Kymographs were analyzed using the Fiji KymographBuilder PlugIn, using three independent experiments and 10-15 time-lapse recordings per condition. Three or four kymographs (containing between 6 and 10 tracks) per recording were generated using the Fiji PlugIn KymographBuilder (RRID for KymographBuilder PlugIn not available, for Fiji software: RRID:SCR_002285). Comovement was evaluated by quantifying the percentage of moving tracks, where moving was defined as a deflection $>20$ degrees from vertical for at least $30 \mathrm{~s}$. Significance was determined by one-way ANOVA with post hoc Tukey (see Fig. $8 A-D$ ).

\section{Results}

\section{CaSR regulates growth in early, BDNF-TrkB-independent chicken nodose neurons}

To address how the CaSR is able to enhance BDNF-mediated growth, we investigated how CaSR signals in isolation. First, we verified that chicken nodose neurons express CaSR but do not express TrkB receptors at early stages of development. It is reported that TrkB levels are very low at Stage 20 to Stage 22 of the Hamburger-Hamilton stages (Hamburger and Hamilton, 1992) and increase by twofold at Stage 23 and sixfold at Stage 24 (Robinson et al., 1996). Using Stage 22 chicken nodose neurons, we found no detectable levels of TrkB in Western blots (Fig. 1A). Since our CaSR antibody did not work in Western blots, we confirmed expression by performing immunocytochemistry and found the CaSR being expressed in Stage 22 nodose neurons (Fig. 
A

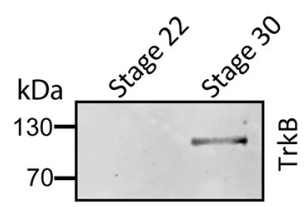

C
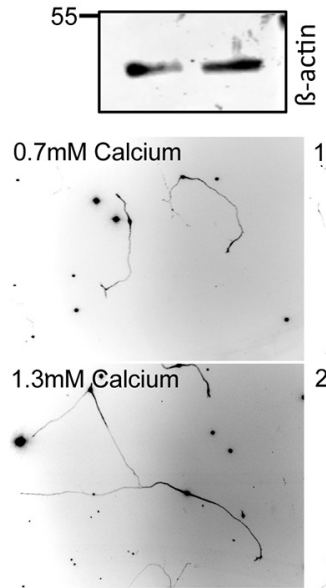

0.7.mM +CaSR Agonist

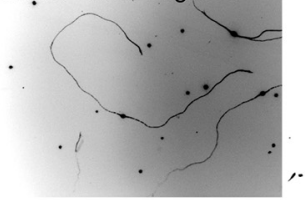

F

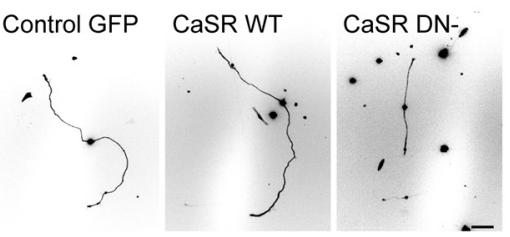

B

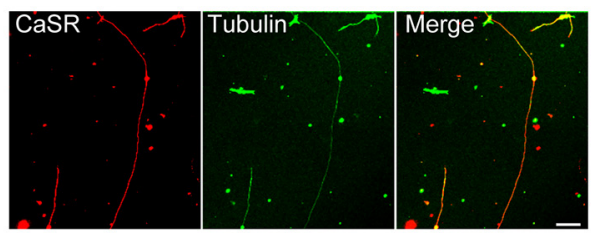

D
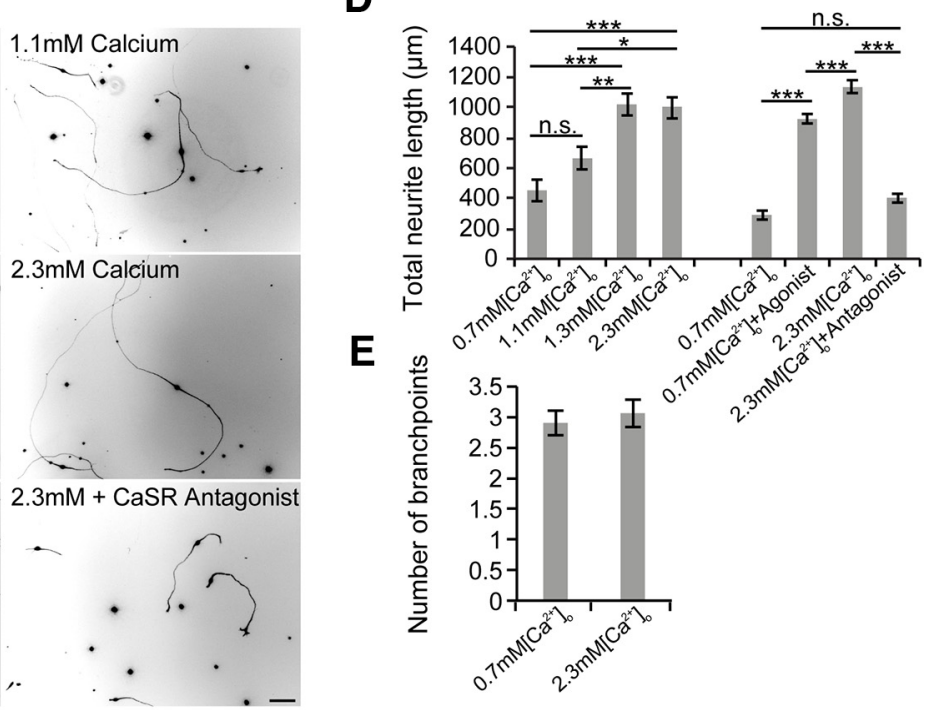

G

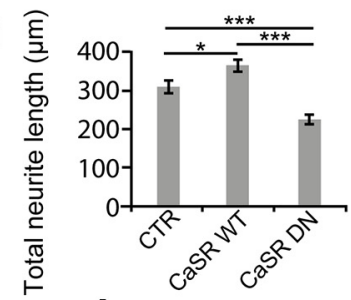

I

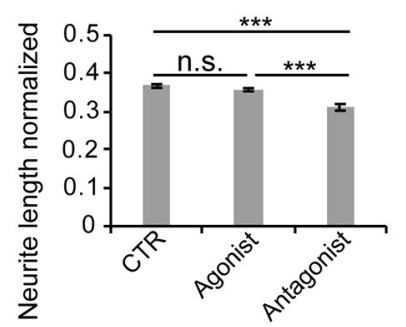

Figure 1. CaSR regulates growth in BDNF-independent nodose neurons. $A, B$, Expression of CaSR and TrkB in Stage 22 nodose neurons using Western blot and immunocytochemistry. C-E, Effects of high extracellular calcium, the CaSR agonist R568 hydrochloride, and the CaSR antagonist Calhex231 on neurite growth and branching in Stage 22 nodose neurons. $F$, G, In vitro effects of CaSR overexpression. $\boldsymbol{H}, \boldsymbol{I}$, Effects to the CaSR agonist R568 hydrochloride and the CaSR antagonist Calhex231 hydrochloride on neurite growth in vivo. Red arrows point to the end of the longest neurite of the nodose ganglion. Red dashed line indicates exemplary measurement of length. An overview of the area is given and ganglia marked in color as follows: pink represents nodose; blue represents petrosal; green represents vestibular; yellow represents trigeminal. Light blue shading represents the area of drug application. Quantification normalized to circumference of midbrain. Scale bar, $100 \mu \mathrm{m}$. Significance was determined by one-way ANOVA with posthoc Tukey. $n=25-60$ images per condition from three or four independent experiments (D, G).DFn $=3, \mathrm{DFd}=641$ (D), DFn $=2, \mathrm{DFd}=209$ (G). $n=3$ or 4 embryos per condition from three independent experiments. $\mathrm{DFn}=2, \mathrm{DFd}=150(I)$. Branch points were analyzed using Student's test to compare $0.7 \mathrm{~mm}$ with $2.3 \mathrm{~mm} \mathrm{DF}=62(\boldsymbol{E}) . n=15-20 \mathrm{images}$ per condition from three independent experiments. Error bar indicates SEM. ${ }^{*} p<0.05,{ }^{* *} p<0.01,{ }^{* * *} p<0.001$. Non-significant differences are indicated as n.s.

1B). Further, assessing neuronal survival during these early stages revealed that neurons do not require BDNF (see Fig. 3C).

Next, we raised the question whether CaSR can regulate axonal growth in BDNF-independent nodose neurons. Previous work reports enhanced growth of mouse SCG neurons using increased levels of extracellular calcium as well as a specific pharmacological CaSR agonist while showing reduced growth in the presence of a specific pharmacological antagonist (Vizard et al., 2008). At Stage 22, where TrkB is not expressed yet, increasing levels of extracellular calcium enhanced neurite growth (Fig. $1 C, D)$. We also found an increase in neurite length in the presence of $0.7 \mathrm{~mm}$ calcium together with the pharmacological CaSR agonist R568 hydrochloride (for calcium concentration, $F_{(3,192)}$ $=15.26$; for agonist and antagonist experiments, $F_{(3,641)}=150.3$, $1.1 \mathrm{~mm}\left[\mathrm{Ca}^{2+}\right]_{\mathrm{o}}$ vs $1.3\left[\mathrm{Ca}^{2+}\right]_{\mathrm{o}}, p=0.007 ; 1.1 \mathrm{~mm}\left[\mathrm{Ca}^{2+}\right]_{\mathrm{o}}$ vs 2.3 $\left[\mathrm{Ca}^{2+}\right]_{\mathrm{o}}, p=0.0138$; for significance, GraphPad Prism 6.0 software does not provide exact $p$ values under a range of $p<0.0001$, post hoc Tukey; Figure $1 C, D)$. Further, we also found reduced 
neurite length in the presence of high extracellular calcium (2.3 $\mathrm{mm}$ ) and the pharmacological CaSR antagonist Calhex231 hydrochloride (Fig. 1C,D). Therefore, activating CaSR enhances neurite growth during early development when neurons are still independent of neurotrophic support.

To test whether CaSR activation induces branching, we quantified branch points per neuron. In all conditions, Stage 22 nodose neurons showed a bipolar morphology with an occasional branch point. We found no differences in branch points between neurons cultured in $0.7 \mathrm{~mm}$ calcium or in $2.3 \mathrm{~mm}$ calcium ( $p=$ 0.599 , unpaired $t$ test; Fig. $1 E$ ). To further investigate whether this is a specific response to CaSR, we transfected neurons with a CaSR-WT (referring to full-length CaSR) and a CaSR-dominant negative (DN) construct and compared neurite length to a GFPtransfected control. When transfecting in vitro, we found that overexpressing CaSR-WT enhanced neurite length compared with GFP control. The R185Q mutation of CaSR has been shown to attenuate the response to high extracellular calcium since the mutation resides within the extracellular domain where it modulates the affinity to extracellular calcium as well as to other agonists (Bai et al., 1996). When overexpressing the CaSR-R185Q construct in nodose neurons, we found a significant decrease in neurite length compared with control and the CaSR-WT construct $\left(F_{(2,209)}=23.671\right.$, GFP vs WT, $p=0.0242$, GFP vs DN and WT vs DN, $p<0.0001$, post hoc Tukey; Fig. $1 F, G)$.

Next, we investigated axon growth effects in vivo. Because of the location of the nodose ganglia, in ovo electroporation proved to be technically challenging. Applying current for electroporation caused heart failure and embryonic death. Therefore, we used a different approach and applied the CaSR agonist R568 hydrochloride and the CaSR antagonist Calhex231 hydrochloride in the area of the nodose at Stage 18 and fixed the embryos at Stage 20. The area of application as well as the location of cranial neurons are shown in Figure $1 \mathrm{H}$. Following whole-mount neurofilament staining, we measured the length of nodose neurons from the hindbrain to the longest neurite (method of measurement is indicated as a dashed line, arrow points to the longest neurite) and normalized the length to the circumference of the midbrain. Using this approach, we found no further increase in length using the CaSR agonist, but a significant decrease in neurite length with the CaSR antagonist $\left(F_{(2,150)}=21.208\right.$, CTR vs antagonist and agonist vs antagonist, $p<0.0001$, post hoc Tukey; Fig. $1 H, I)$.

\section{At Stage 22, CaSR activates p-Akt and localizes to Rab7- and Lamp1-positive endosomes}

Following the observation that CaSR regulates BDNF-independent neurite growth, we examined the downstream signaling pathway. Commonly described pathways in regulating axonal growth are the PI3-kinase-Akt-GSK3 pathway or the Mek-Erk pathway (Chao, 2003; Yoshimura et al., 2005; Zhou et al., 2008). Therefore, we dissected early Stage 22 chick nodose neurons, placed them in culture medium containing low $(0.7 \mathrm{~mm})$ and high $(2.3$ $\mathrm{mm}$ ) levels of extracellular calcium in the presence of specific PI3-kinase, GSK3, and Mek inhibitors, and measured their response on neurite growth. We found that early chick nodose neurons, when cultured in $2.3 \mathrm{~mm}$ calcium, respond solely to the PI3-kinase inhibitor LY294002 with decreased growth but do not respond to the GSK3 and Mek inhibitors BIO and PD98059, respectively (Fig. $2 A, B$ ). Further, the CaSR was reported to signal through Mek-Erk in different cell types (Holstein et al., 2004; Brennan et al., 2013; Vizard et al., 2015; Mizumachi et al., 2017), a response we did not observe in Stage 22 chicken nodose neu- rons. $\left(F_{(3,142)}=14.262,2.3 \mathrm{~mm}\left[\mathrm{Ca}^{2+}\right]_{\mathrm{o}}\right.$ vs $2.3 \mathrm{~mm}\left[\mathrm{Ca}^{2+}\right]_{\mathrm{o}}+\mathrm{LY}$ $p<0.0001$, post hoc Dunnett in $2.3 \mathrm{~mm}\left[\mathrm{Ca}^{2+}\right]_{o}$; Fig. $\left.2 A, B\right)$.

To confirm and to place this response specifically downstream of the CaSR, we stimulated these neurons with the allosteric CaSR agonist (in $0.7 \mathrm{~mm}$ calcium) and antagonist (in $2.3 \mathrm{~mm}$ calcium) and performed Western blots against p-Akt, p-GSK3 $\alpha$ Tyr279, and p-GSK3 $\beta$ Tyr2 16 and p-GSK $3 \beta$ Ser9. We found that indeed p-Akt was increased after activating CaSR but strongly decreased in the presence of the CaSR antagonist $\left(F_{(4,11)}=34.708,0.7 \mathrm{~mm}\right.$ $\left[\mathrm{Ca}^{2+}\right]_{\mathrm{o}} \mathrm{vs} 15^{\prime} 0.7 \mathrm{~mm}\left[\mathrm{Ca}^{2+}\right]_{\mathrm{o}}+$ agonist and $5^{\prime}, 15^{\prime}$, and $30^{\prime} 2.3$ $\mathrm{mm}\left[\mathrm{Ca}^{2+}\right]_{\mathrm{o}}+$ antagonist vs $15^{\prime} 0.7 \mathrm{~mm}\left[\mathrm{Ca}^{2+}\right]_{\mathrm{o}}+$ agonist, $p<$ 0.0001 , post hoc Tukey; Fig. 2C-E). Surprisingly, but in line with the observation that the GSK3 inhibitor BIO had no effect on neurite growth, we did not find phosphorylation on GSK3 $\alpha$ Tyr279, p-GSK3 $\beta$ Tyr216, or GSK3 $\beta$ Ser9. This result was unexpected since the PI3-kinase-Akt pathway has been reported to be upstream of GSK3 $\beta$ Ser9 (Yoshimura et al., 2005; Zhou et al., 2008; Bellon et al., 2010; Burk et al., 2017a), suggesting an uncoupled mechanism (Fig. 2C).

Because trafficking and signaling of receptors are tightly linked (Wu et al., 2001; Barford et al., 2017; Burk et al., 2017a), we next investigated the trafficking route of CaSR. Literature reports that CaSR is endocytosed and trafficked through Rab11-recycling endosomes, as well as being degraded through late endosomes (Holstein et al., 2004; Reyes-Ibarra et al., 2007; Zhuang et al., 2012; Ray, 2015). We performed colocalization experiments of CaSR with different endosomal compartments using antibodies against CaSR as well as EEA1, Rab11, Rab7, and Lamp1 to represent early, recycling, and late endosomes as well as lysosomes. When stimulated with the CaSR agonist in medium containing $0.7 \mathrm{~mm}$ calcium, we found high colocalization of activated CaSR with Rab7- and Lamp1-positive late endosomes/lysosomes $\left(F_{(3,588)}=89.412\right.$, except EEA1 vs Rab11, $p=0.9996$, all other conditions compared in post hoc Tukey, $p<0.0001$; Fig. $2 F, G$ ). In all, our data show that CaSR regulates neurotrophinindependent growth by activating Akt.

\section{Late, BDNF-dependent neurite growth is enhanced by CaSR activation}

Next, we studied the effect of CaSR activation in Stage 30 nodose neurons, which are dependent on BDNF for growth and survival (Robinson et al., 1996). In Stage 30 nodose neuron cultures, CaSR and TrkB are expressed in neurons but not in nonneuronal cells (Fig. $3 A$ ).

Further, we found that CaSR and TrkB show colocalizing puncta in nodose neurons (Fig. 3B). As previously reported (Vogel and Davies, 1991; Robinson et al., 1996), Stage 30 nodose neurons depend on BDNF for survival from Stage 24 onwards $\left(F_{(7,98)}=26.336\right.$, Stage 25 no factor vs BDNF and Stage 30 no factor vs BDNF significance under a range of $p<0.0001$, post hoc Sidak; Fig. $3 C$ ). Since we found that CaSR regulates BDNFindependent growth, we raised the question whether CaSR activation could still regulate neurite growth in the absence of BDNF. To investigate this, neurons were plated in the presence of caspase inhibitors to block apoptosis (Gavaldà et al., 2009) and plated in either $0.7 \mathrm{~mm}$ or $2.3 \mathrm{~mm}$ calcium. Interestingly, at these later stages when neurons depend on BDNF for survival, CaSR activation alone failed to induce neurite growth (Fig. $3 D, E$ ). We then examined the effect of BDNF in the presence of low levels of extracellular calcium $(0.7 \mathrm{~mm})$ where the CaSR should be minimally active (Vizard et al., 2008). Here, BDNF-induced neurite growth significantly increased compared with neurons cultured in the presence of caspase inhibitors (Fig. $3 D, E$ ). However, plat- 
A

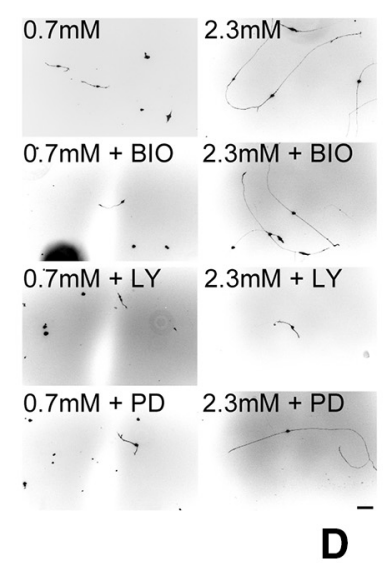

C

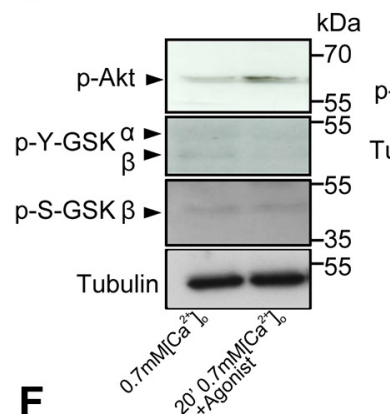

D
B

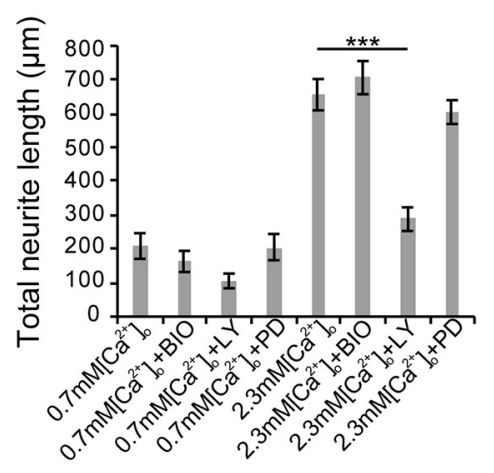

E

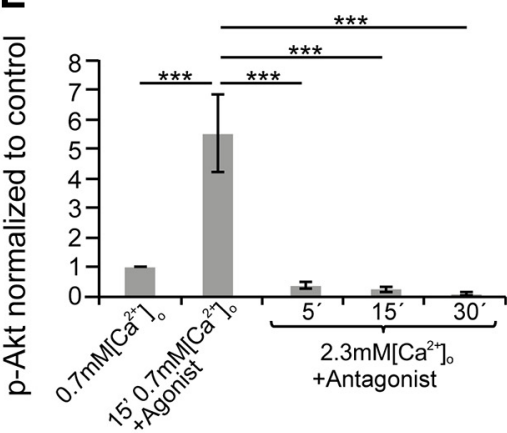

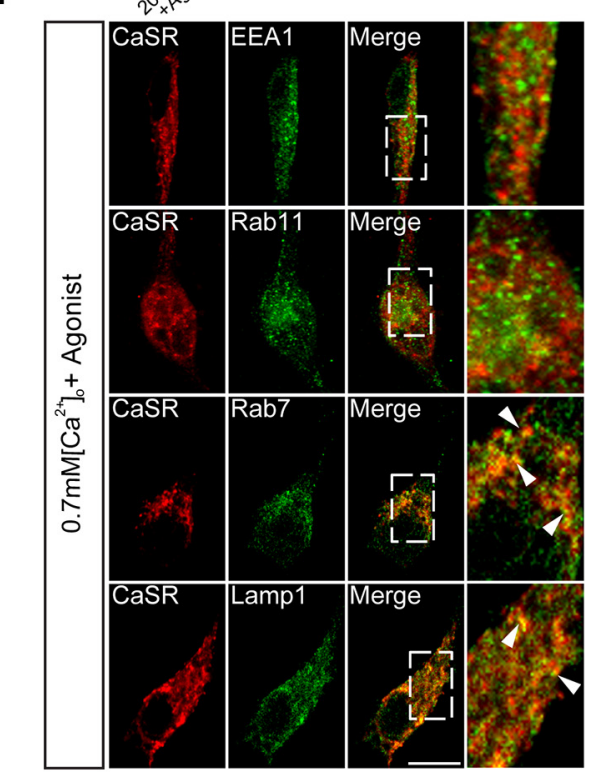

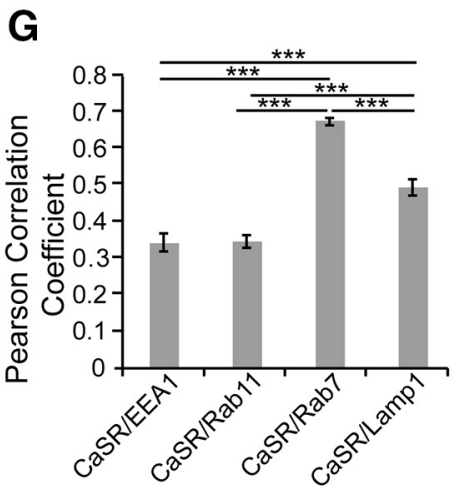

Figure 2. At Stage 22, CaSR signals through Akt and routes into late endosomes. $A, B$, Effects of inhibitors for GSK3 (BIO), PI3-kinase (LY), and Mek (PD) on neurite length in the presence of 0.7 or $2.3 \mathrm{~mm}$ extracellular calcium. $\boldsymbol{C}, \boldsymbol{D}$, Western blots for p-Akt and p-GSK3 after stimulation with the CaSR agonist and antagonist. $\boldsymbol{E}$, Quantitation of p-Akt normalized to tubulin and control condition $(0.7 \mathrm{~mm}$ calcium normalized to each loading control). $\boldsymbol{F}, \boldsymbol{G}$, Colocalization of CaSR with endosomal markers. Arrows point to colocalizing puncta. $N=4-6$ independent experiments for Western blots. Scale bars: $\boldsymbol{A}, 100 \mu \mathrm{m} ; \boldsymbol{F}, 10 \mu \mathrm{m}$. Significance was determined by one-way ANOVA with post hoc Dunnett compared with control ( $0.7 \mathrm{~mm}$ calcium + agonist) $(\boldsymbol{B})$ or Tukey $(\boldsymbol{E}, \boldsymbol{G}) . n=25-60$ images per condition from four independent experiments $(\boldsymbol{B}, \boldsymbol{G}) \cdot \mathrm{DFn}=3, \mathrm{DFd}=142(\boldsymbol{B}), \mathrm{DFn}=3, \mathrm{DFd}=588(\boldsymbol{G}) \cdot n=$ three independent experiments. DFn $=4, \mathrm{DFd}=11(\boldsymbol{E})$. Error bar indicates SEM. ${ }^{* * *} p<0.001$.

ing neurons in the presence of BDNF together with $2.3 \mathrm{~mm}$ calcium or in $0.7 \mathrm{~mm}$ and in the CaSR agonist, further increased neurite length significantly compared with BDNF alone (Fig. $3 D, E)$. In contrast, plating neurons in $2.3 \mathrm{~mm}$ calcium containing BDNF and the CaSR antagonist Calhex 231 reduced neurite length below the level of BDNF in $0.7 \mathrm{~mm}$ extracellular calcium $\left(F_{(5,10271)}=230.187\right.$, for $0.7 \mathrm{~mm}\left[\mathrm{Ca}^{2+}\right]_{\mathrm{o}}+$ BDNF vs $2.3 \mathrm{~mm}$ $\left[\mathrm{Ca}^{2+}\right]_{\mathrm{o}}+$ antagonist, $p=0.0009$, all other conditions compared in post hoc Tukey are under a range of $p<0.0001$; Fig. $3 D, E)$. In addition to neurite length, we analyzed branching of these neurons. We found that Stage 30 nodose neurons, when cultured in $2.3 \mathrm{~mm}$ calcium and $10 \mathrm{ng} / \mathrm{ml} \mathrm{BDNF}$, had more branch points compared with $0.7 \mathrm{~mm}$ calcium control cultures containing 10 $\mathrm{ng} / \mathrm{ml}$ BDNF. Also, when neurons were cultured in $2.3 \mathrm{~mm}$ calcium, $10 \mathrm{ng} / \mathrm{ml} \mathrm{BDNF}$ and CaSR antagonist, branch points were significantly reduced compared with neurons cultured in $0.7 \mathrm{mM}$, calcium with $10 \mathrm{ng} / \mathrm{ml} \mathrm{BDNF}$ and the CaSR agonist $(0.7 \mathrm{~mm}$ $\left[\mathrm{Ca}^{2+}\right]_{\mathrm{o}}$ vs $2.3 \mathrm{~mm}\left[\mathrm{Ca}^{2+}\right]_{\mathrm{o}}$ and $0.7 \mathrm{~mm}\left[\mathrm{Ca}^{2+}\right]_{\mathrm{o}}+$ agonist vs 2.3 

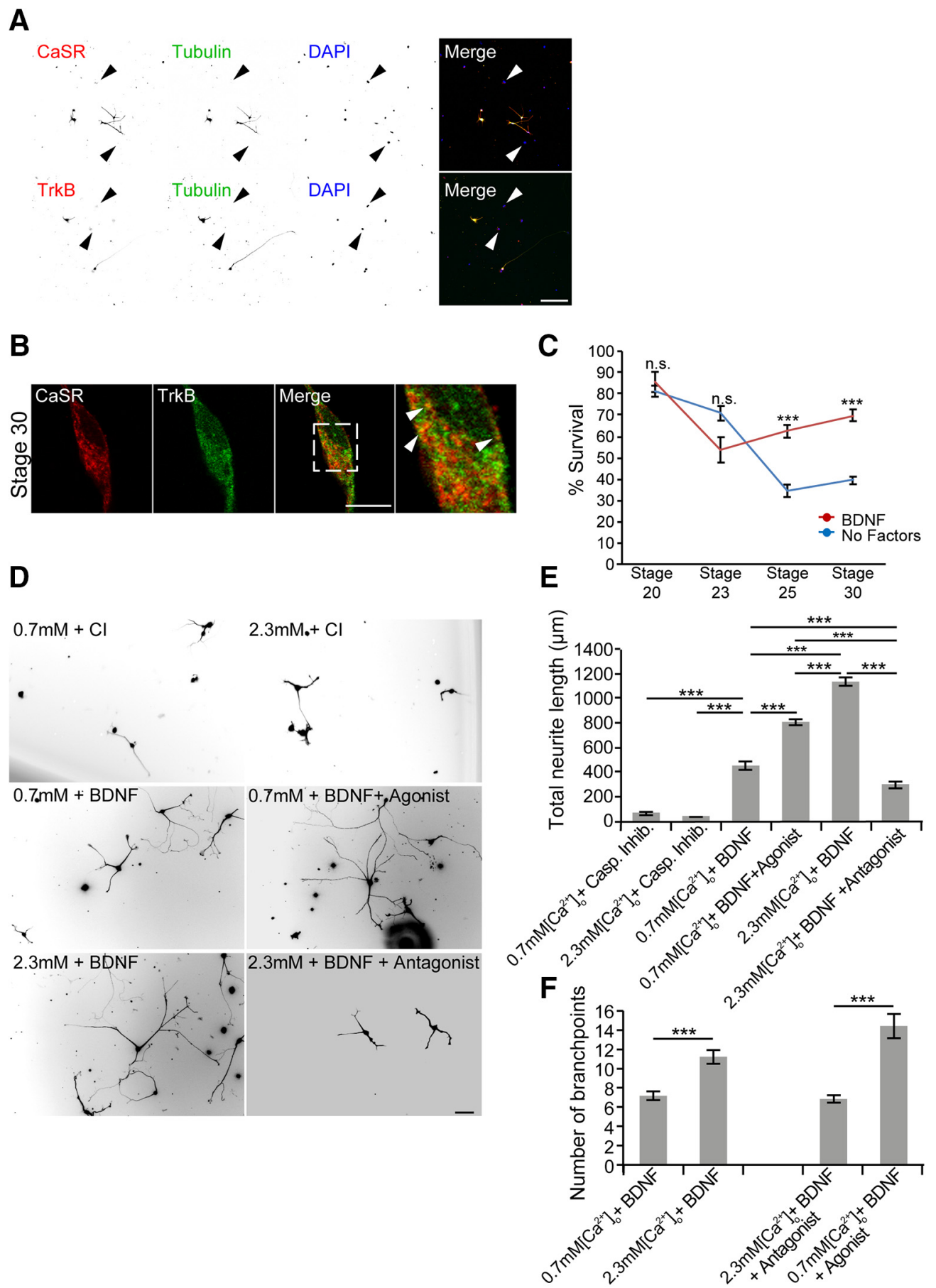

Figure 3. CaSR regulates growth in BDNF-dependent nodose neurons. $A$, Expression of CaSR and TrkB in Stage 30 nodose neurons but not in non-neuronal cells using immunocytochemistry. Arrows point to non-neuronal cells. $\boldsymbol{B}$, CaSR and TrkB colocalizing in Stage 30 nodose neurons using immunocytochemistry. Arrows point to colocalizing puncta. $\boldsymbol{C}$, Neuronal survival over development in the presence and absence of BDNF. D, Effects of high extracellular calcium, the CaSR agonist R568 hydrochloride, the CaSR antagonist Calhex231, and BDNF on neurite growth in Stage 30 nodose neurons. (I, Caspase inhibitors. E, Quantitation of neuronal length as shown in D.F, Quantitation of branch points in $0.7 \mathrm{~mm}$ calcium, $2.3 \mathrm{~mm}$ calcium (together with BDNF), or with the CaSR agonist (in 0.7 mu calcium) and the CaSR antagonist (in $2.3 \mathrm{~mm}$ calcium, both in the presence of BDNF). Scale bars: $A, C, 100 \mu \mathrm{m} ; \boldsymbol{B}, 10 \mu \mathrm{m}$. Significance was determined by one-way ANOVA with posthoc Sidak. DFn $=7$, DFd $=$ $98(\boldsymbol{C})$ or Tukey $(\boldsymbol{E}) \cdot n=25-60$ images per condition from five independent experiments. DFn $=5, \mathrm{DFd}=1271$. Branch points were analyzed using Student's $t$ test to compare $0.7 \mathrm{~mm}$ with $2.3 \mathrm{~mm}$ and $2.3 \mathrm{~mm}+$ CaSR antagonist with $0.7 \mathrm{~mm}+$ CaSR agonist DF $=75(\boldsymbol{F})$. Error bar indicates SEM. ${ }^{* * *} p<0.001$. Non-significant differences are indicated as n.s.

$\mathrm{mm}\left[\mathrm{Ca}^{2+}\right]_{\mathrm{o}}+$ antagonist $p<0.0001$, unpaired $t$ test; Fig. $\left.3 F\right)$. These results indicate that during a time point where neurons innervate their targets in vivo (Davies and Lindsay, 1985; Davies et al., 1986; Davies, 1989; Vogel and Davies, 1991; Robinson et al., 1996), CaSR enhances BDNF-mediated neurite length and branching from Stage 30 but fails to regulate growth when stimulated in isolation. This result is in line with previous reports by
Vizard et al. (2008) where the authors show that the CaSR enhances NGF-mediated neurite growth.

At Stage 30, CaSR activates p-tyrosine GSK3 and localizes to Rab7 and Lamp1-positive endosomes

Surprisingly, at Stage 30, we found the downstream signaling cascade of CaSR changed after the onset of BDNF dependence. 
A

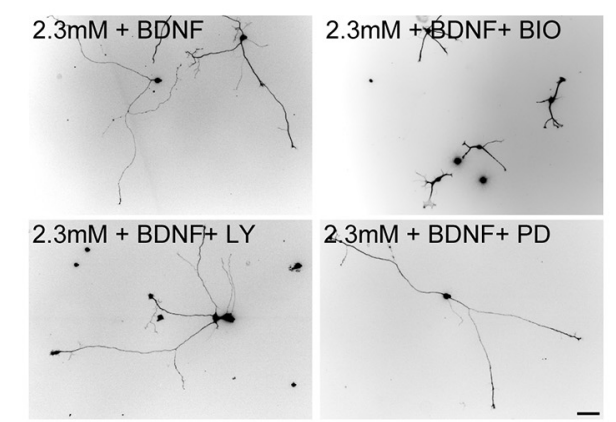

B

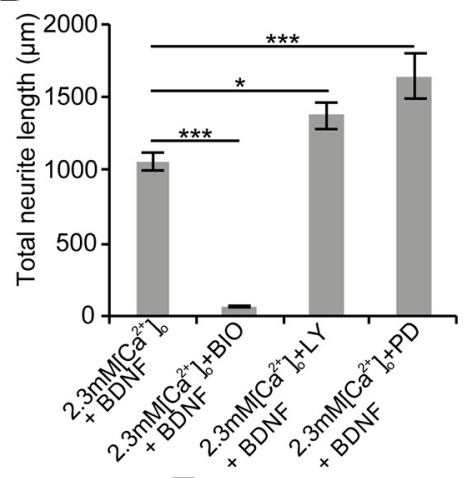

C

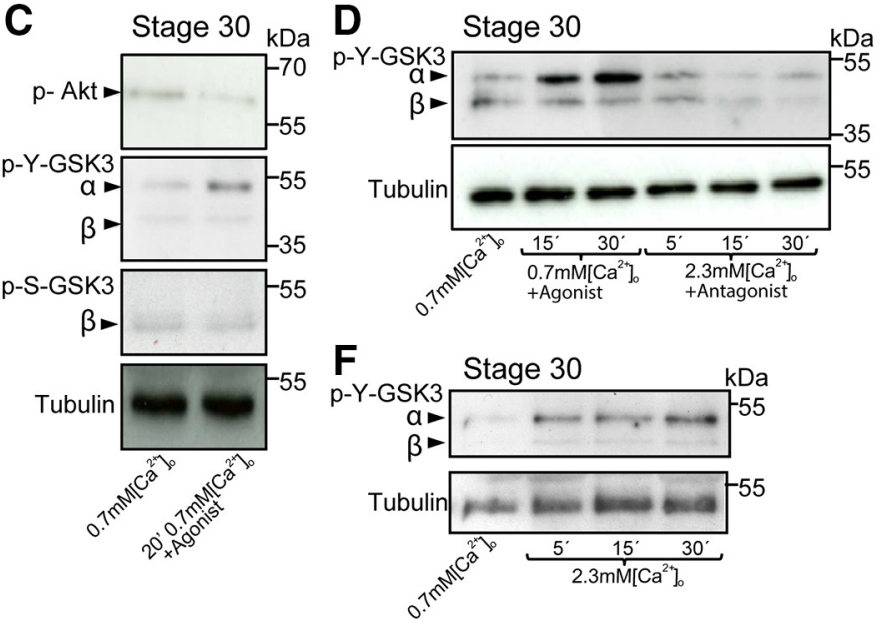

$E$

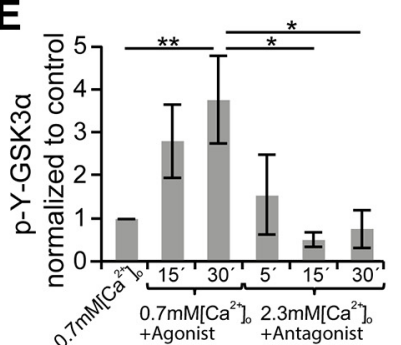

Figure 4. At Stage 30, CaSR signals through GSK3.A, B, Effects and quantitation of inhibitors for GSK3, PI3-kinase, and Mek (BI0, LY, and PD, respectively) on neurite length in the presence of 2.3 mm extracellular calcium. C, D, Western blots for p-Akt and p-GSK3 after stimulation with the CaSR agonist or antagonist. $\boldsymbol{E}$, Quantitation of p-GSK3 normalized to tubulin and control condition (0.7 mm calcium normalized to each loading control). $\boldsymbol{F}$, Western blot for p-GSK3 Tyr279 $(\alpha)$ and Tyr216 $(\beta)$ after stimulation with $2.3 \mathrm{~mm}$ calcium. $N=4-6$ independent experiments for Western blots. Scale bar: $\boldsymbol{A}, 100 \mu \mathrm{m}$. Significance was determined by one-way ANOVA with post hoc Tukey $(\boldsymbol{E})$ or Dunnett $(\boldsymbol{B})$ compared with control ( $2.3 \mathrm{~mm}+\mathrm{BDNF}) . n=25-60 \mathrm{images}$ per condition from four independent experiments. DFn $=3, \mathrm{DFd}=131(\boldsymbol{B}), \mathrm{DFn}=5, \mathrm{DFd}=21(\boldsymbol{E})$. Error bar indicates SEM. ${ }^{*} p<0.05,{ }^{* *} p<0.01,{ }^{* * *} p<0.001$.

When plating neurons in the presence of $2.3 \mathrm{~mm}$ calcium containing $10 \mathrm{ng} / \mathrm{ml} \mathrm{BDNF}$ as well as in the presence of PI3-kinase, GSK3, or Mek inhibitors, we found a different response compared with Stage 22 BDNF-independent neurons. Unlike at Stage 22, the PI3-kinase inhibitor LY294002 as well as Mek inhibition using PD98059 showed an increase on neurite growth, whereas blocking GSK3 signaling inhibited neurite growth significantly $\left(F_{(3,131)}=76.104,2.3 \mathrm{~mm}\left[\mathrm{Ca}^{2+}\right]_{\mathrm{o}}+\right.$ BDNF vs $2.3 \mathrm{~mm}\left[\mathrm{Ca}^{2+}\right]_{\mathrm{o}}+$ BDNF + LY, $p=0.0129$, all other conditions compared with control under a range of $p<0.0001$, post hoc Dunnett; Fig. $4 A, B$ ).

We confirmed this result using biochemistry. For this experiment, we grew neurons for $24 \mathrm{~h}$ in plating medium containing 10 $\mathrm{ng} / \mathrm{ml}$ of BDNF to ensure growth and survival. The next day, neurons were starved in "starving medium" adjusted to $0.7 \mathrm{~mm}$ without BDNF for $20 \mathrm{~min}$. Subsequently, neurons were stimulated in starving medium containing the allosteric CaSR agonist (in $0.7 \mathrm{~mm}$ calcium) or antagonist (in $2.3 \mathrm{~mm}$ calcium), and lysates were analyzed by Western blots. Stimulating Stage 30 nodose neurons with CaSR agonist did not lead to phosphorylation of Akt (Fig. 4C1). In contrast, stimulation of CaSR by the CaSR agonist as well as $2.3 \mathrm{~mm}$ calcium increased phosphorylation on GSK3 $\alpha$ Tyr279 (Fig. 4C2, E, F) but not on GSK3 $\beta$ Ser9 (Fig. 4C3). This phosphorylation of Tyr279 could be reversed by adding the CaSR antagonist in the presence of $2.3 \mathrm{~mm}$ calcium $\left(F_{(5,21)}=\right.$ 4.677, $0.7 \mathrm{mM}\left[\mathrm{Ca}^{2+}\right]_{\mathrm{o}}$ vs $30^{\prime} 0.7 \mathrm{mM}\left[\mathrm{Ca}^{2+}\right]_{\mathrm{o}}+$ agonist, $p=$ $0.0097,30^{\prime} 0.7 \mathrm{~mm}\left[\mathrm{Ca}^{2+}\right]_{\mathrm{o}}+$ agonist vs $15^{\prime} 2.3 \mathrm{~mm}\left[\mathrm{Ca}^{2+}\right]_{\mathrm{o}}+$ antagonist, $p=0.0231,30^{\prime} 0.7 \mathrm{mM}\left[\mathrm{Ca}^{2+}\right]_{\mathrm{o}}+$ agonist vs $30^{\prime} 2.3$ $\mathrm{mm}\left[\mathrm{Ca}^{2+}\right]_{\mathrm{o}}+$ antagonist, $p=0.0412$, post hoc Tukey; Fig. $4 D, E)$.

Because the signaling cascade completely changed in BDNFdependent nodose neurons, we assessed whether this was linked to a change in the trafficking path of CaSR. We repeated colocalization experiments using antibodies for the CaSR and EEA1, Rab11, Rab7, and Lamp1 to represent early, recycling, and late endosomes, respectively. We found that at BDNF-dependent stages, activated CaSR still localized to late, Rab7, and Lamp-1 positive endosomes $\left(F_{(3,429)}=168.384\right.$, except for Rab7 vs Lamp1, $p=0.9105$; all other conditions are under a range of $p<$ 0.0001 , post hoc Tukey; Fig. $5 C, D$ ) whereas in nonstimulated conditions, CaSR localized mainly to Lamp1-positive compartments $\left(F_{(3,86)}=10.804\right.$, Lamp1 vs EEA1, $p=0.0010$, Lamp1 vs Rab11, $p=0.0004$, Lamp1 vs Rab7, $p<0.0001$, post hoc Tukey; Fig. $5 A, B)$.

\section{CaSR and TrkB colocalize in late Rab7-positive endosomes}

Because the change in signaling was not facilitated by a change in localization of CaSR to endosomes, we wanted to determine whether the change in signaling of CaSR was dependent on TrkB. Therefore, we first investigated the localization of TrkB in Stage 30 chicken nodose neurons. We performed immunostainings and colocalization experiments using antibodies against TrkB with antibodies against EEA1, Rab11, Rab7, or Lamp1. We found 
A

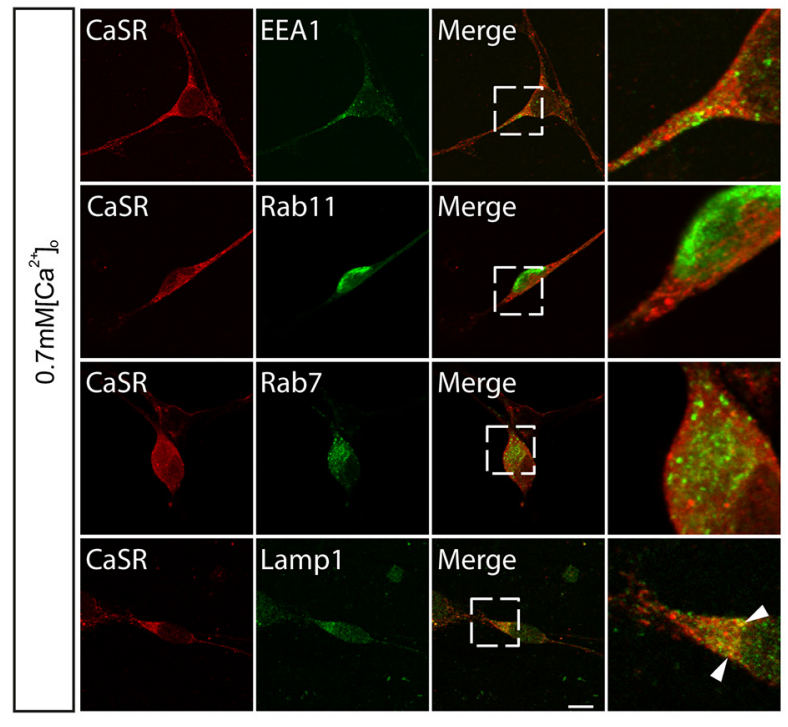

C

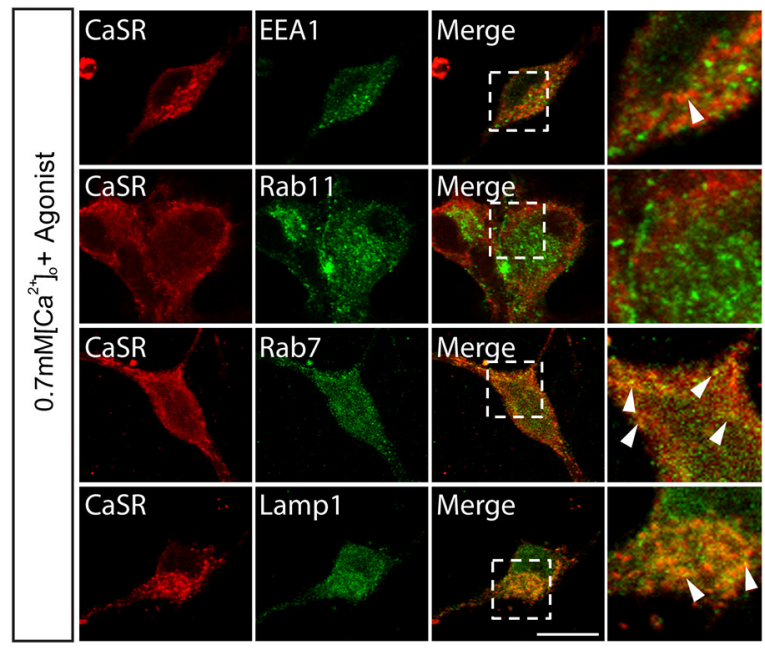

B

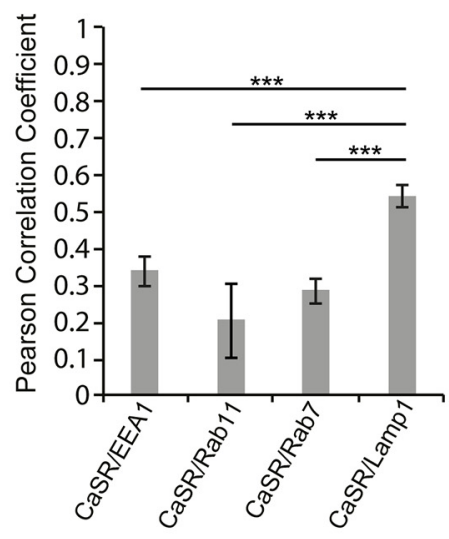

D

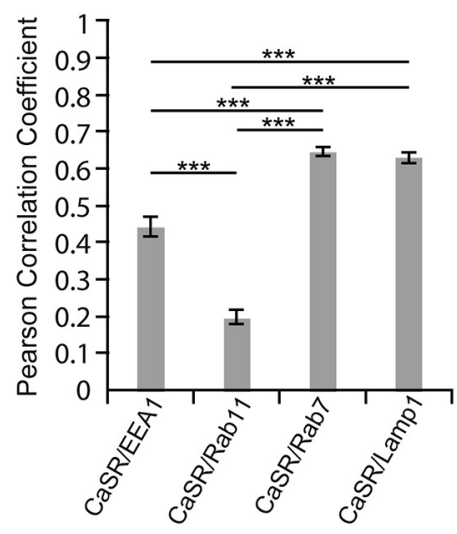

Figure 5. Activated CaSR localizes to Rab7-positive endosomes. A, B, Colocalization and quantitation of CaSR with endosomal markers EEA1, Rab11, Rab7, and Lamp1 in nonstimulated conditions. C, D, Colocalization and quantitation of CaSR with endosomal markers Rab7 and Lamp1 in the presence of the CaSR agonist. Arrows point to colocalizing puncta. Scale bar, $10 \mu m$. Significance was determined by one-way ANOVA with post hoc Tukey. $n=15-30$ images per condition from three independent experiments. DFn $=3, \mathrm{DFd}=86(\boldsymbol{B}), \mathrm{DFn}=3, \mathrm{DFd}=429(\boldsymbol{D})$. Error bar indicates SEM. ${ }^{* * *} p<0.001$.

that, when starved of BDNF and calcium, TrkB showed low localization to all endosomal compartments but highest colocalization to recycling endosomes $\left(F_{(3,89)}=15.788\right.$, Rab11 vs all other conditions, $p<0.0001$, post hoc Tukey; Fig. $6 A, B)$. However, in the presence of BDNF in $0.7 \mathrm{~mm}$ calcium, TrkB routed from early into late, Rab7-positive endosomes $\left(F_{(3,145)}=43.783\right.$, EEA1 vs Lamp1, $p=0.0104$, EEA1 vs Rab11, $p=0.0001$, EEA1 vs Rab7, $p<0.0001$, Rab7 vs Rab11, $p<0.0001$ and Rab7 vs Lamp1, $p<$ 0.0001 , post hoc Tukey; Fig. $6 C, D)$. The routing of activated TrkB into Rab7 endosomes has been reported by other groups (Kuruvilla et al., 2004; Deinhardt et al., 2006; Burk et al., 2017b). Further, Trk receptors start their downstream signaling from the endosomal compartment to which they localize (Wu et al., 2001; Kuruvilla et al., 2004; Saxena et al., 2005; Zhou et al., 2012; Barford et al., 2017). To evaluate whether CaSR and TrkB colocalize in endosomal compartments, we performed colocalization experiments between CaSR, TrkB, and the late endosomal markers Rab7 or Lamp1. We found that CaSR and TrkB show a high level of colocalization with each other. Also, both receptors show high colocalization with Rab7-positive endosomes. However, colocalization between CaSR and Lamp1 as well as TrkB and Lamp1 was reduced compared with $\operatorname{Rab} 7\left(F_{(5,180)}=30.241\right.$, TrkB/Rab7 vs TrkB/Lamp1 and CaSR/Rab7 vs CaSR/Lamp1, $p<0.0001$, post hoc Sidak; Fig. $6 E, F)$. These results indicate that at later developmental stages, CaSR and TrkB traffic within the same Rab7positive endosomal compartments.

\section{CaSR and TrkB coimmunoprecipitate and cotraffic}

In an event where changes in signaling of CaSR were dependent on TrkB, we hypothesized that both receptors have to be in close proximity and physically interact. As mentioned, using immunofluorescence in Stage 30 chicken nodose neurons, we found colabeled puncta for CaSR and TrkB (Figs. 3B, 6E,F).

To determine whether both receptors interact physically, we overexpressed CaSR-WT-GFP or CaSR-DN-GFP together with either RFP or TrkB-RFP in HEK293 cells and performed RFP- 

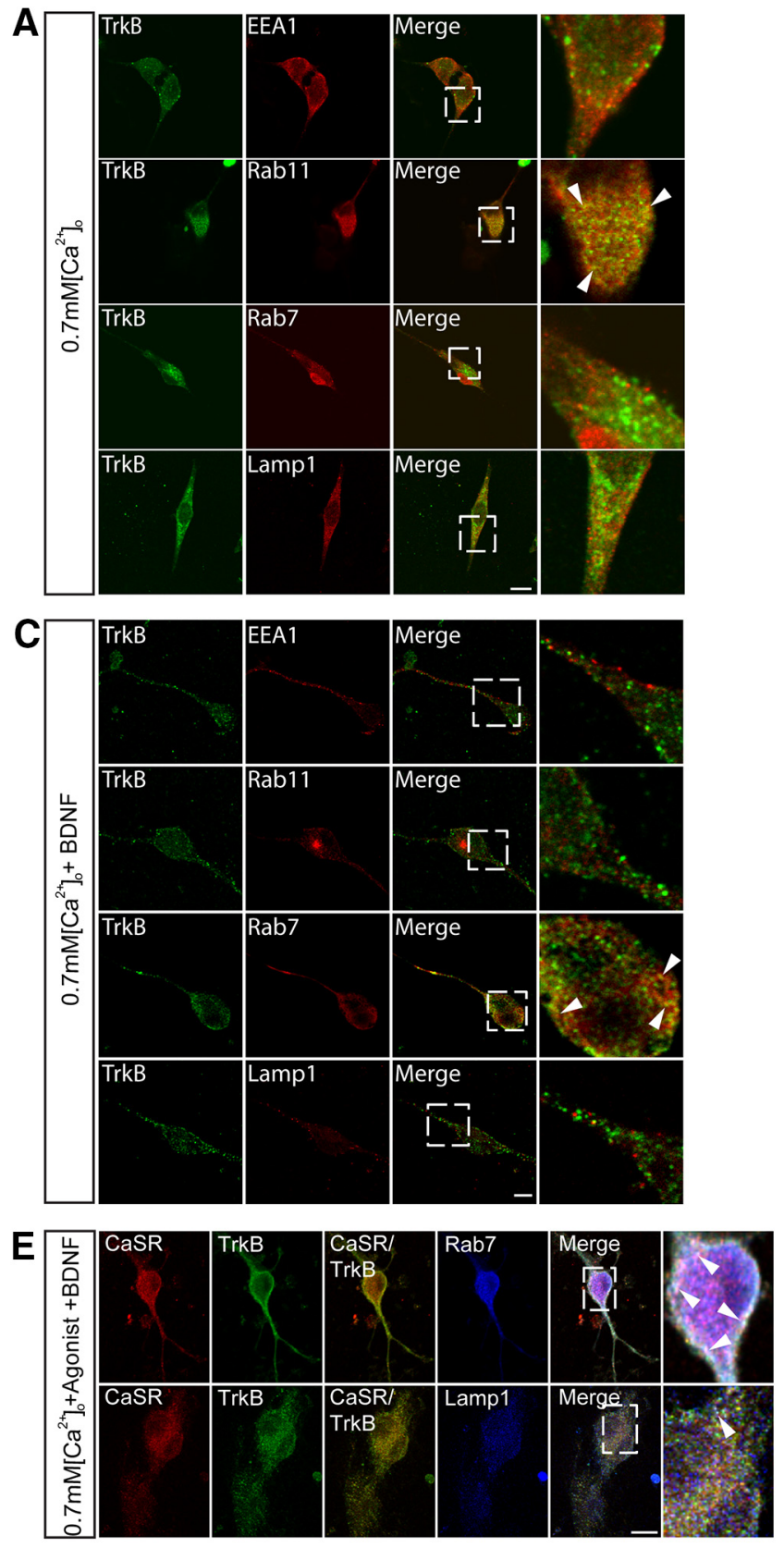

Figure 6. Activated TrkB localizes in Rab7-positive endosomes where it colocalizes with CaSR. A, B, Colocalization and quantitation of TrkB with endosomal markers EEA1, Rab11, Rab7, and Lamp1 in nonstimulated conditions. C, D, Localization and quantitation of TrkB and the endosomal markers EEA1, Rab11, Rab7, and Lamp1 in the presence of BDNF. $\boldsymbol{E}$, $\boldsymbol{F}$, Colocalization and quantitation of CaSR and TrkB as well as with endosomal markers Rab7 and Lamp1. Arrows point to colocalizing puncta. Scale bar, $10 \mu \mathrm{m}$. Significance was determined by one-way ANOVA with post hoc Tukey test $(\boldsymbol{B}, \boldsymbol{D})$ or Sidak test $(\boldsymbol{F}) . n=15-30$ images per condition from three independent experiments. DFn $=3, \mathrm{DFd}=89(\boldsymbol{B}), \mathrm{DFn}=3, \mathrm{DFd}=145(\boldsymbol{D}), \mathrm{DFn}=5, \mathrm{DFd}=180(\boldsymbol{F})$. Error bar indicates SEM. ${ }^{*} p<0.05,{ }^{* * *} p<0.001$.

Trap coimmunoprecipitation. Both CaSR-WT and CaSR-DN coimmunoprecipitated with TrkB but not with control (i.e., IgGcoated beads or when CaSR-GFP was coexpressed with RFP only) (Fig. 7A). The CaSR-DN construct R185Q has its mutation in the extracellular domain where it modulates the affinity to extracellular calcium as well as to other agonists, which could explain the interaction of R185Q with TrkB.

In a GFP-Trap reverse coimmunoprecipitation, we overexpressed CaSR-GFP with TrkB-RFP and tested whether interactions were ligand-dependent. We again found an interaction
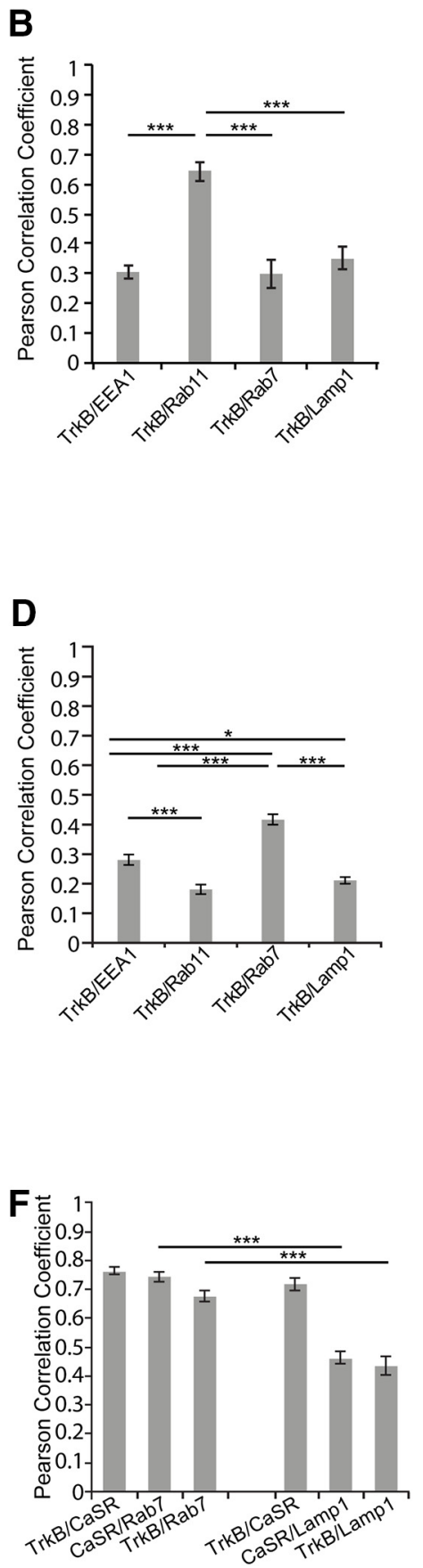

between CaSR and TrkB, and this interaction was ligandindependent (Fig. 7B).

To verify the interaction in neuronal cells, we overexpressed CaSR-WT and CaSR-DN constructs in N1E-115 cells and performed RFP-Trap coimmunoprecipitation. Also, in these cells, we found interaction of TrkB with both CaSR-WT and CaSR-DN (Fig. 7C).

To investigate whether both receptors cotraffic, we overexpressed a CaSR-GFP and a TrkB-RFP construct in MEFs and kept them either in nonstimulated $(0.7 \mathrm{mM})$ or stimulated conditions 
A
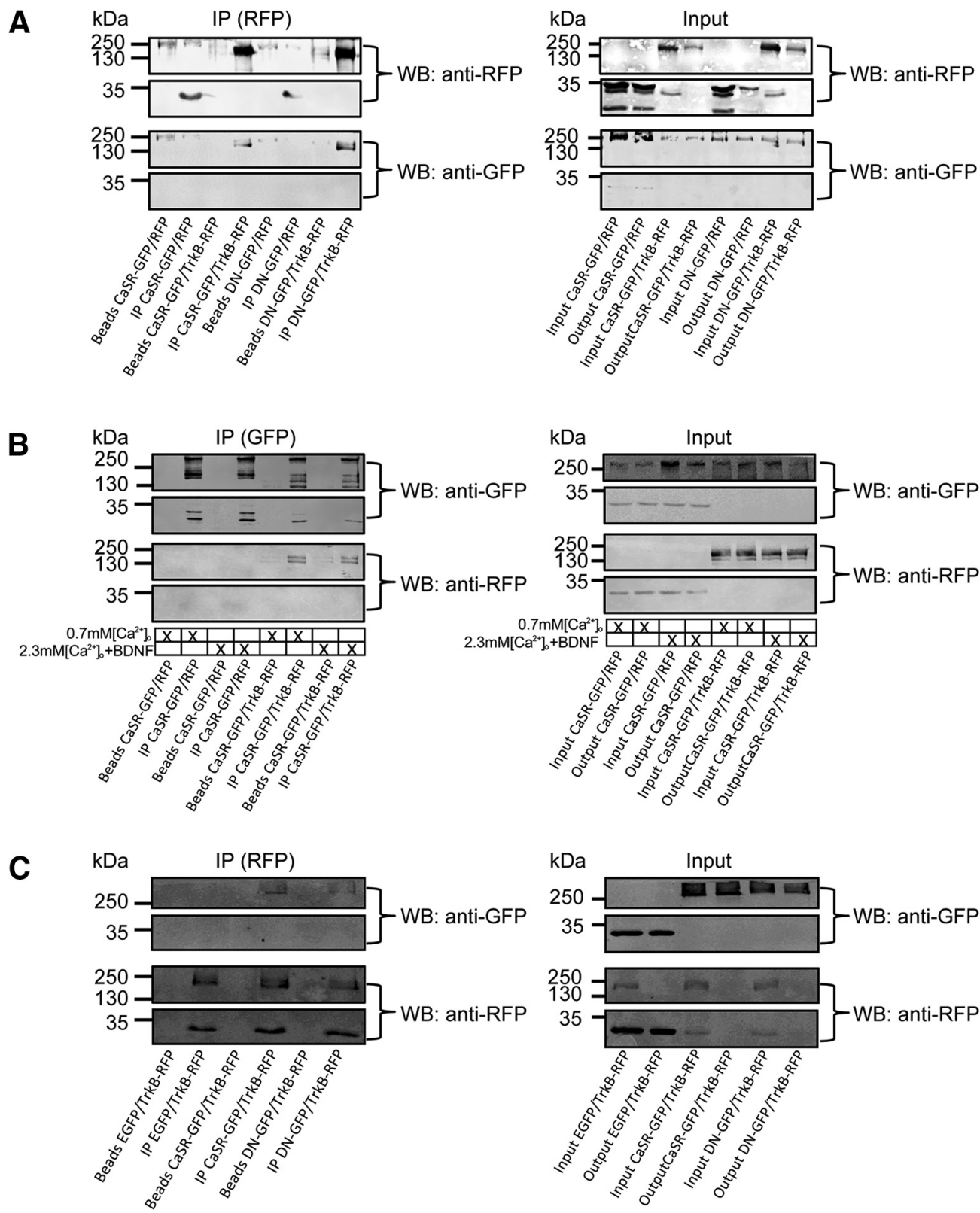

Figure 7. CaSR coimmunoprecipitates with TrkB. A, CaSR and CaSR DN coimmunoprecipitate with TrkB in HEK293 cells. B, Interaction of CaSR and TrkB is ligand-independent. C, CaSR and CaSR DN interact with TrkB in neuronal N1E-115 cells.

(0.7 mm calcium together with the CaSR agonist, BDNF, or both) and first performed colocalization experiments. Using this approach, we found that CaSR and TrkB colocalize in all above conditions to similar extent (ANOVA nonsignificant; Fig. $8 D$, Pearson's colocalization index ranging from 0.73 to 0.8 ), further supporting our finding that colocalization was ligandindependent.

We then asked whether both receptors cotraffic and whether this cotrafficking was dependent on receptor activation. To test this, we performed TIRF microscopy. Our experimental set up included the following conditions: nonstimulated $(0.7 \mathrm{~mm}$ calcium), $0.7 \mathrm{~mm}$ calcium + CaSR agonist R568 hydrochloride, 0.7 $\mathrm{mm}$ calcium + BDNF, and $0.7 \mathrm{~mm}$ calcium + BDNF + R568 hydrochloride.

Despite the colocalization and interaction in all conditions (Fig. $8 D$ ), we found different behaviors for cotrafficking: in control conditions ( $0.7 \mathrm{~mm}$ calcium), the CaSR and TrkB had colocalizing tracks but minimal comovement (Fig. 8A,B). When we stimulated one of the two receptors, by either applying BDNF or the CaSR agonist R568 hydrochloride, comovement increased significantly compared with control and was equal between both receptors (Fig. $8 A, B$ ). Coactivation of both receptors by applying BDNF and CaSR agonist R568 hydrochloride further increased cotrafficking significantly compared with activation of CaSR or TrkB alone $\left(F_{(3,332)}=\right.$ 24.1, $0.7 \mathrm{mM}\left[\mathrm{Ca}^{2+}\right]_{\mathrm{o}}$ vs $0.7 \mathrm{mM}\left[\mathrm{Ca}^{2+}\right]_{\mathrm{o}}+\mathrm{BDNF}, p=0.0025$, $0.7 \mathrm{~mm}\left[\mathrm{Ca}^{2+}\right]_{\mathrm{o}}$ vs $0.7 \mathrm{~mm}\left[\mathrm{Ca}^{2+}\right]_{\mathrm{o}}+$ agonist, $p=0.0011,0.7$ $\mathrm{mm}\left[\mathrm{Ca}^{2+}\right]_{\mathrm{o}}$ vs $0.7 \mathrm{~mm}\left[\mathrm{Ca}^{2+}\right]_{\mathrm{o}}+$ agonist + BDNF, $p<$ $0.0001,0.7 \mathrm{~mm}\left[\mathrm{Ca}^{2+}\right]_{\mathrm{o}}+\mathrm{BDNF}$ vs $0.7 \mathrm{~mm}\left[\mathrm{Ca}^{2+}\right]_{\mathrm{o}}+$ agonist $+\mathrm{BDNF}, p<0.0001$, and $0.7 \mathrm{~mm}\left[\mathrm{Ca}^{2+}\right]_{\mathrm{o}}+$ agonist vs $0.7 \mathrm{~mm}$ $\left[\mathrm{Ca}^{2+}\right]_{\mathrm{o}}+$ agonist + BDNF, $p<0.0001$, post hoc Tukey; Fig. $8 A, B)$. Next, we overexpressed the R185Q CaSR construct together with TrkB and performed cotrafficking analysis. In- 


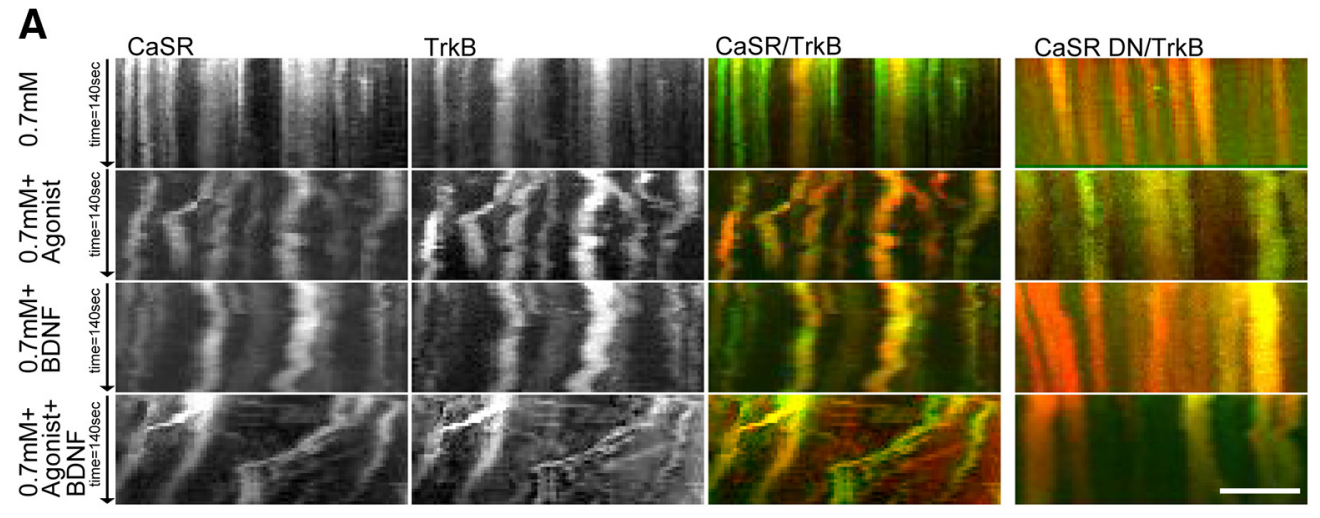

B

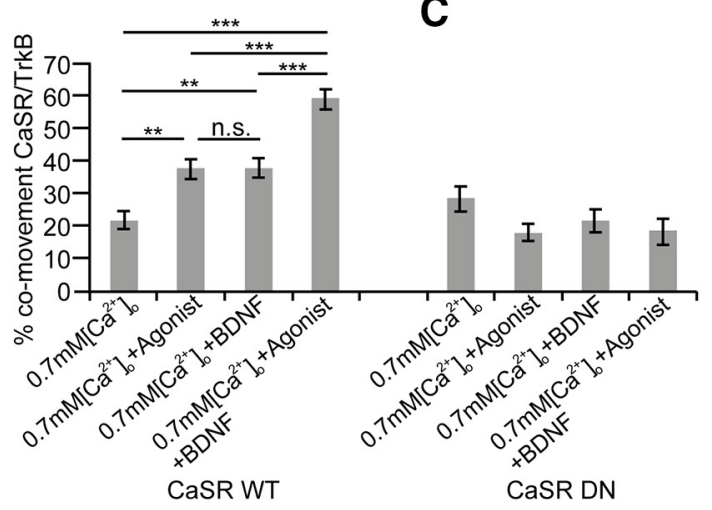

D

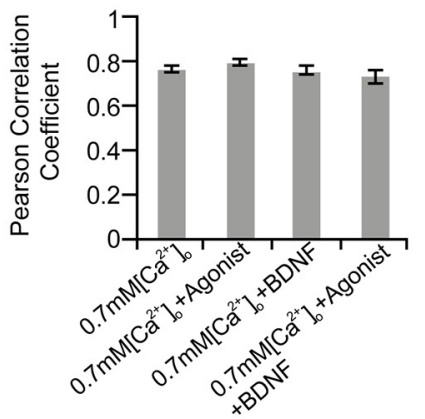

Figure 8. CaSR traffics together with TrkB. A, Kymographs of cotrafficking CaSR or CaSR-DN and TrkB in unstimulated and stimulated conditions from TIRF time-lapse images of MEFs cotransfected with CaSR-GFP or CaSR-DN-GFP and TrkB-RFP. B, Quantitation of comovement of CaSR and TrkB. Both, CaSR and TrkB when stimulated individually are able to increase comovement. This comovement further increases when both receptors are active simultaneously. $A$, C, Comovement decreases when TrkB is overexpressed with CaSR DN R185R. D, Colocalization of CaSR and TrkB under nonstimulated and stimulated conditions. Scale bar, $10 \mu \mathrm{m}$. Significance was determined by one-way ANOVA with post hoc Tukey. $n=25$ images per condition from three independent experiments. DFn $=3, \mathrm{DFd}=332(\boldsymbol{B}), \mathrm{DFn}=3, \mathrm{DFd}=199$ (C). Error bar indicates SEM. ${ }^{* *} p<0.01$, ${ }^{* * *} p<0.001$. Non-significant differences are indicated as $n . s$.

terestingly, using the CaSR DN construct, we did not find an increase in cotrafficking in any of the conditions (ANOVA nonsignificant; Fig. $8 \mathrm{~A}, \mathrm{C})$.

\section{CaSR and TrkB regulate growth through coactivation of GSK3}

As mentioned earlier, receptor trafficking from endosomes and their downstream signaling are tightly linked (Dobrowolski and De Robertis, 2011; Pálfy et al., 2012; Harrington and Ginty, 2013). Since we found that CaSR and TrkB localize to the same endosomal compartments, coimmunoprecipitate and cotraffic, we raised the question whether enhanced neurite growth is modulated because both receptors activate the same signaling node.

In Figures $4 C, D, F$ and $9 A, B$, we found that CaSR activation leads to a significant increase in phosphorylation on GSK3 $\alpha$ $\operatorname{Tyr} 279\left(F_{(3,14)}=3.550,0.7 \mathrm{~mm}\left[\mathrm{Ca}^{2+}\right]_{\mathrm{o}}\right.$ vs $30^{\prime} 0.7 \mathrm{~mm}\left[\mathrm{Ca}^{2+}\right]_{\mathrm{o}}+$ agonist, $p=0.036$, post hoc Dunnett; Fig. $9 A, B)$. Therefore, we tested whether TrkB activation through BDNF can also cause phosphorylation on GSK $3 \alpha$ Tyr279 and GSK3 $\beta$ Tyr216. Interestingly, stimulation of Stage 30 nodose neurons with BDNF in 0.7 $\mathrm{mm}$ calcium significantly increased phosphorylation on GSK3 $\alpha$ Tyr279 after $30 \mathrm{~min}$ as well as on GSK3 $\beta$ Tyr2 16 at 15 and $30 \mathrm{~min}$ $\left(F_{(3,12)}=4.862\right.$, GSK3 $\alpha$ Tyr279: $0.7 \mathrm{~mm}\left[\mathrm{Ca}^{2+}\right]_{\mathrm{o}}$ vs $30^{\prime} 0.7 \mathrm{~mm}$ $\left[\mathrm{Ca}^{2+}\right]_{\mathrm{o}}+\mathrm{BDNF}, p=0.008 ; F_{(3,12)}=7.984$, GSK3 $\beta$ Tyr216: 0.7 $\mathrm{mm}\left[\mathrm{Ca}^{2+}\right]_{\mathrm{o}}$ vs $15^{\prime} 0.7 \mathrm{~mm}\left[\mathrm{Ca}^{2+}\right]_{\mathrm{o}}+\mathrm{BDNF}, p=0.0226$ and 0.7 $\mathrm{mm}\left[\mathrm{Ca}^{2+}\right]_{\mathrm{o}}$ vs $30^{\prime} 0.7 \mathrm{~mm}\left[\mathrm{Ca}^{2+}\right]_{\mathrm{o}}+$ BDNF $p=0.0016$, post hoc Dunnett; Figure $9 C, D)$. Further, BDNF also activated the previously reported GSK3 $\beta$ Ser9 pathway (Yoshimura et al., 2005), showing that BDNF is able to target GSK3 on three phosphorylation sites in nodose neurons (Fig. 9C).

Next, we hypothesized that coactivation of CaSR and TrkB would potentially lead to an additive effect on GSK3 $\alpha$ Tyr279 and GSK3 $\beta$ Tyr216, given the fact that both receptors phosphorylate these residues when stimulated individually. To test this, we starved Stage 30 nodose neurons in $0.7 \mathrm{~mm}$ calcium and applied either the CaSR agonist R568 hydrochloride or BDNF + R568 hydrochloride to cultured neurons.

When CaSR and TrkB were activated simultaneously, phosphorylation on GSK3 $\alpha$ Tyr279 significantly increased at 5, 15, and $30 \mathrm{~min}\left(F_{(4,19)}=3.623,0.7 \mathrm{mM}\left[\mathrm{Ca}^{2+}\right]_{\mathrm{o}}\right.$ vs $30^{\prime} 0.7 \mathrm{~mm}$ $\left[\mathrm{Ca}^{2+}\right]_{\mathrm{o}}+$ agonist, $p=0.049,0.7 \mathrm{mM}\left[\mathrm{Ca}^{2+}\right]_{\mathrm{o}}$ vs $15^{\prime} 0.7 \mathrm{~mm}$ $\left[\mathrm{Ca}^{2+}\right]_{\mathrm{o}}+\mathrm{BDNF}+$ agonist, $p=0.0326,0.7 \mathrm{mM}^{2}\left[\mathrm{Ca}^{2+}\right]_{\mathrm{o}}$ vs $30^{\prime}$ $0.7 \mathrm{~mm}\left[\mathrm{Ca}^{2+}\right]_{\mathrm{o}}+\mathrm{BDNF}+$ agonist, $p=0.0151$, post hoc Dunnett; Fig. 9E, F). However, given our hypothesis that we would potentially see an additive effect, we were surprised that there was no increase in phosphorylation on GSK3 $\alpha$ Tyr279 compared with agonist stimulation only and that phosphorylation on GSK3 $\beta$ Tyr216 disappeared (Fig. 9E,F). To show that BDNF was added to the cultures and that it was functional, we stripped and reprobed the membrane with an anti-GSK $3 \beta$ Ser9 antibody. Control and agonist lanes confirmed the absence of BDNF while in 5,15 , and $30 \mathrm{~min}$ an increase in p-GSK3 Ser9 appeared due to BDNF stimulation (Fig. 9E). This result suggests that coactivation of CaSR and TrkB changes the downstream target of TrkB from GSK3 Tyr279/216 and Ser9 to GSK3 Ser9 phosphorylation only. 
A

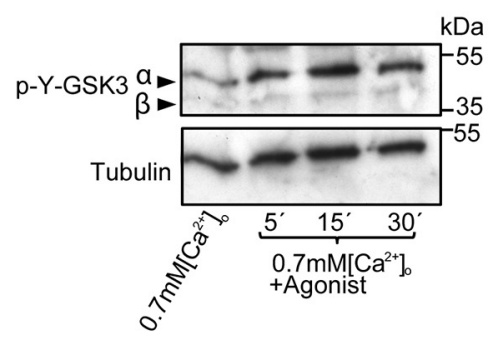

C

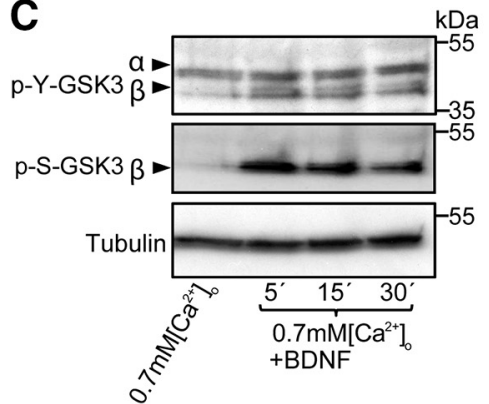

E

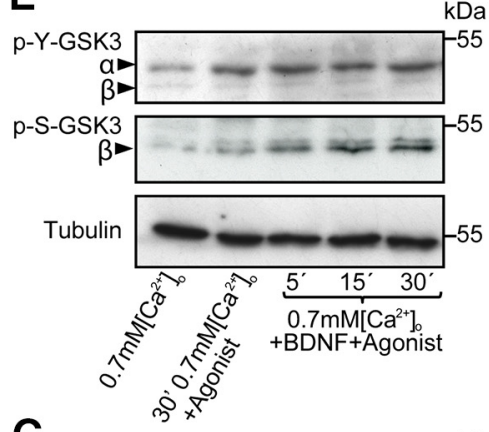

G

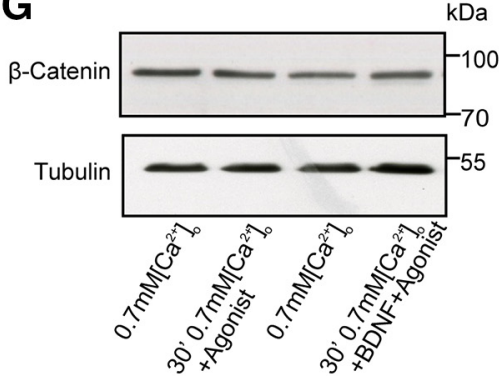

B
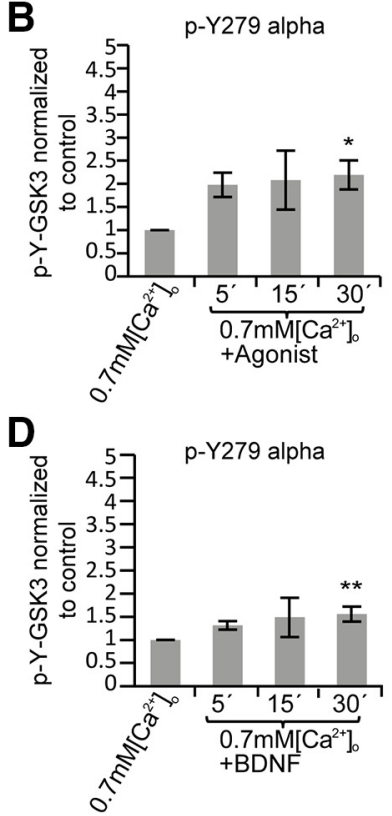

F

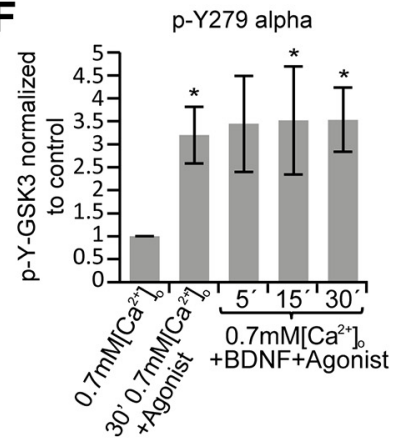

p-Y216 beta

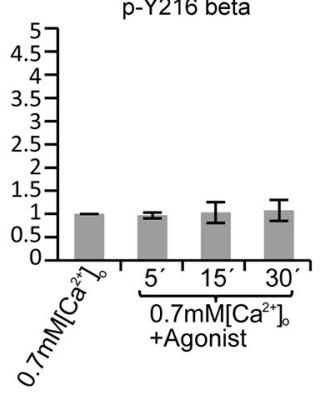

p-Y216 beta

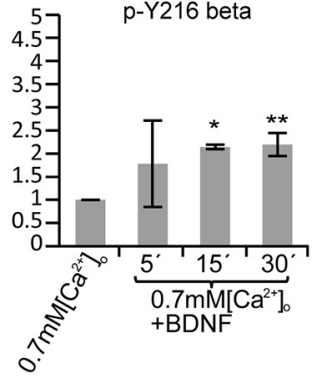

p-Y216 beta

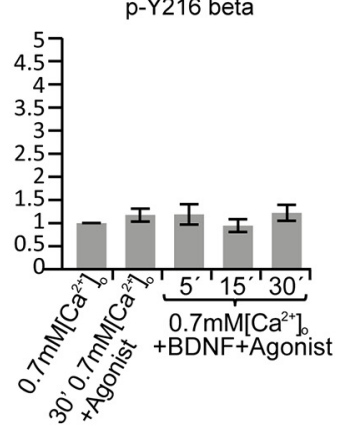

Figure 9. CaSR and TrkB activate GSK3. A, Western blot of p-GSK3 Tyr279 $(\alpha)$ and Tyr216 $(\beta)$ after stimulation with the CaSR agonist R568 hydrochloride. $\boldsymbol{B}$, Quantitation of $p$-GSK3 Tyr normalized to tubulin and $0.7 \mathrm{~mm}$ control. C, Western blot of p-GSK3 Tyr279, Tyr216, and Ser9 after stimulation with BDNF. D, Quantitation of p-GSK3 Tyr normalized to tubulin and $0.7 \mathrm{~mm}$ control. $\boldsymbol{E}$, Western blot of p-GSK3 Tyr279, Tyr216, and Ser9 after stimulation with the CaSR agonist R568 hydrochloride, BDNF, or both. $\boldsymbol{F}$, Quantitation of p-GSK3 Tyr normalized to tubulin and $0.7 \mathrm{~mm}$ control. $\mathbf{G}$, Western blot of $\beta$-catenin after stimulation with the CaSR agonist or CaSR agonist ${ }^{+}$BDNF in 0.7 mM calcium. $N=4-6$ independent experiments. Significance was determined by one-way ANOVA with post hoc Dunnett compared with control (0.7 mm calcium) DFn $=3, \mathrm{DFd}=14(\boldsymbol{B}), \mathrm{DFn}=3, \mathrm{DFd}=12(\boldsymbol{D}), \mathrm{DFn}=4, \mathrm{DFd}=18$ $(\boldsymbol{F})$. Error bar indicates SEM. ${ }^{*} p<0.05,{ }^{* *} p<0.01$.

Because phosphorylation on GSK3 Tyr residues has been reported to increase the activity of GSK3 and phosphorylation on GSK3 Ser9 to decrease the activity (Cross et al., 1995; Hartigan and Johnson, 1999; Lesort et al., 1999; Yoshimura et al., 2005), these results indicate that GSK3 is kept in a cycling state, which potentially affects downstream targets of GSK3.

To test whether other pathways are involved, we also tested whether the $\beta$-catenin pathway, which has been reported to interact with GSK3 and regulate axon growth (Lu et al., 2004), got activated when stimulated with the CaSR agonist and BDNF. In this experiment, we observed no changes following CaSR or TrkB activation, suggesting no involvement of the $\beta$-catenin pathway (Fig. 9G).

\section{Activation of GSK3 through CaSR phosphorylates Tau}

To link activation of GSK3 to neurite growth, we tested whether CaSR activates Tau. To test this, we starved Stage 30 nodose neurons in $0.7 \mathrm{~mm}$ calcium and applied the CaSR agonist for 5 and 15 min as well as the CaSR antagonist for 5, 15, and $30 \mathrm{~min}$. Western blots against $\mathrm{p}$-Tau revealed a significant increase in Tau activation after 15 min of CaSR stimulation and a strong decrease in phosphorylation in the presence of CaSR antagonist $\left(F_{(5,26)}=\right.$ 
A
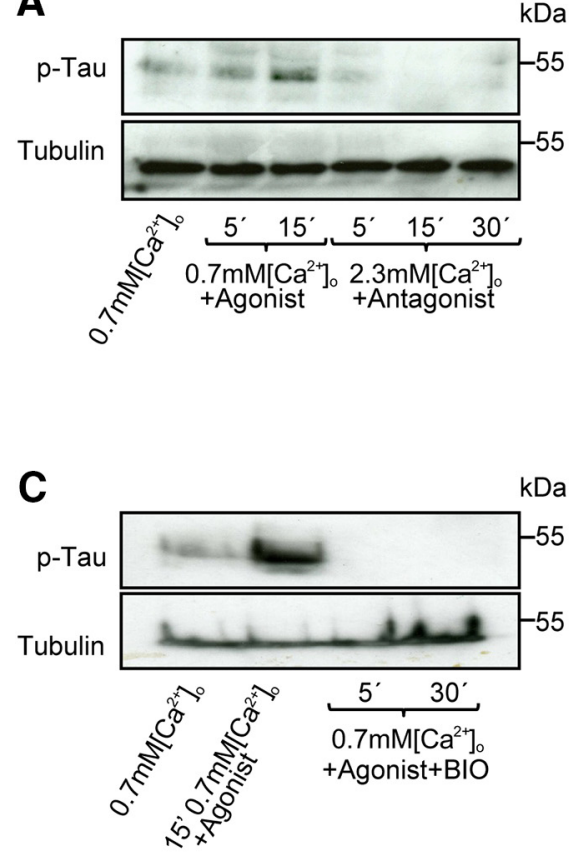

E

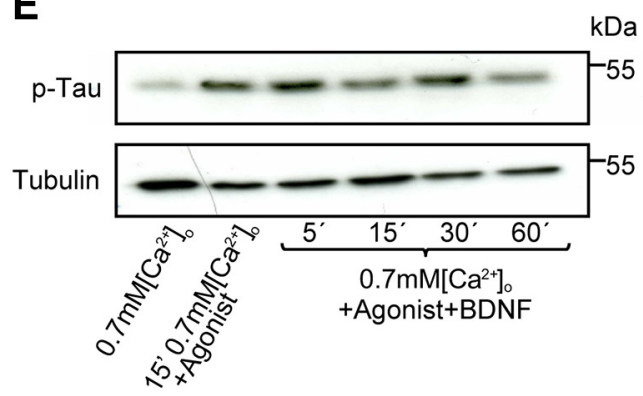

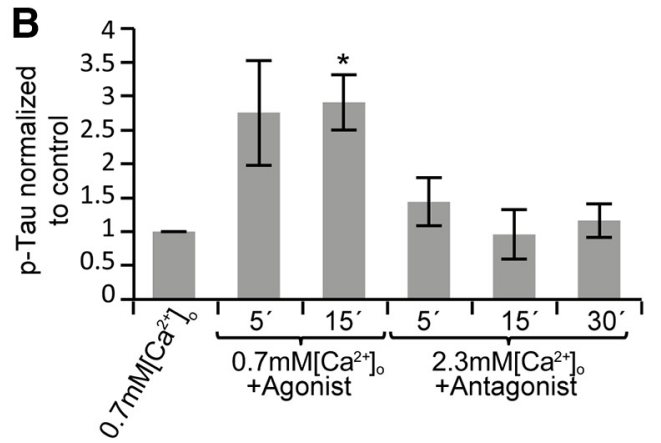

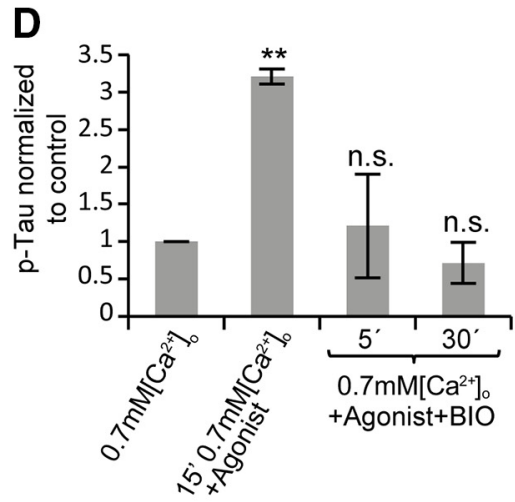

$\mathbf{F}$

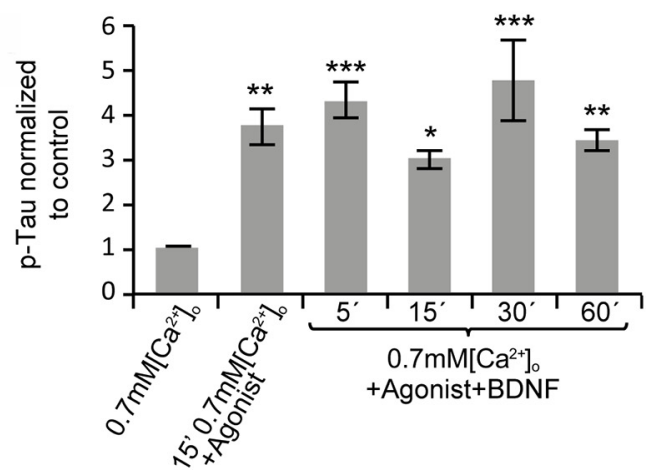

Figure 10. Coactivation of CaSR and TrkB cycles phosphorylation state of Tau. $\boldsymbol{A}$, Western blot of p-Tau after stimulation with R568 hydrochloride in $0.7 \mathrm{~mm}$ for 5 and $15 \mathrm{~min}$ and Calhex 231 in 2.3 $\mathrm{mm}$ calcium for 5, 15, and $30 \mathrm{~min}$. B, Quantitation of p-Tau normalized to tubulin and $0.7 \mathrm{~mm}$ control. C, Western blot of p-Tau after stimulation with R568 hydrochloride in $0.7 \mathrm{~mm}$ calcium or R568 hydrochloride in $0.7 \mathrm{~mm}$ calcium ${ }^{+}$BIO (GSK3 inhibitor). D, Quantitation of p-Tau normalized to tubulin and $0.7 \mathrm{~mm}$ control. $\boldsymbol{E}$, Western blot of p-Tau after a time course stimulation with the CaSR agonist R568 hydrochloride and CaSR agonist R568 ${ }^{+}$BDNF. F, Quantitation of p-Tau normalized to tubulin and $0.7 \mathrm{~mm}$ control. $N=4-6$ independent experiments. Significance was determined by one-way ANOVA with post hoc Dunnett compared with control $\left(0.7 \mathrm{~mm}\right.$ calcium), DFn $=5, \mathrm{DFd}=26(\boldsymbol{B}), \mathrm{DFn}=3, \mathrm{DFd}=18(\boldsymbol{D}), \mathrm{DFn}=5, \mathrm{DFd}=18(\boldsymbol{F})$. Error bar indicates $\mathrm{SEM}$. ${ }^{*} p<0.05$, ${ }^{* *} p<0.01,{ }^{* * *} p<0.001$. Non-significant differences are indicated as n.s.

3.015, $0.7 \mathrm{~mm}\left[\mathrm{Ca}^{2+}\right]_{\mathrm{o}}$ vs $15^{\prime} 0.7 \mathrm{~mm}\left[\mathrm{Ca}^{2+}\right]_{\mathrm{o}}+$ agonist, $p=$ 0.0291 , post hoc Dunnett; Fig. $10 A, B)$. To verify that this activation was downstream of GSK3, we also applied the GSK3 inhibitor BIO to neurons cultured in $0.7 \mathrm{~mm}$ calcium plus the CaSR agonist. Indeed, we found a decrease in $\mathrm{p}$-Tau after blocking $\operatorname{GSK} 3\left(F_{(3,18)}=7.112,0.7 \mathrm{~mm}\left[\mathrm{Ca}^{2+}\right]_{\mathrm{o}}\right.$ vs $15^{\prime} 0.7 \mathrm{~mm}\left[\mathrm{Ca}^{2+}\right]_{\mathrm{o}}+$ agonist, $p=0.0015$, post hoc Dunnett; Fig. $10 C, D)$. Since we hypothesized that GSK3 is regulated in a cycling way between an active and an inactive state through CaSR and TrkB, we speculated that Tau should show fluctuations in its phosphorylation state. We tested this by performing a time course on $\mathrm{p}$-Tau where we used a $0.7 \mathrm{~mm}$ control, $0.7 \mathrm{~mm}$ together with R568 hydrochloride or $0.7 \mathrm{~mm}+\mathrm{R} 568$ hydrochloride $+\mathrm{BDNF}$ for $5,15,30$, and 60 minutes. Indeed, we found that, compared with $0.7 \mathrm{~mm}$ control, Tau was significantly phosphorylated, but we found a cy- cling behavior between the stimulated conditions $\left(F_{(5,18)}=\right.$ 8.042 , all conditions were compared with $0.7 \mathrm{~mm}\left[\mathrm{Ca}^{2+}\right]_{\mathrm{o}}$ for $15^{\prime}$ $0.7 \mathrm{mM}\left[\mathrm{Ca}^{2+}\right]_{\mathrm{o}}+$ agonist, $p=0.0026,5^{\prime} 0.7 \mathrm{mM}\left[\mathrm{Ca}^{2+}\right]_{\mathrm{o}}+$ agonist + BDNF, $p=0.0004,15^{\prime} 0.7 \mathrm{mM}\left[\mathrm{Ca}^{2+}\right]_{\mathrm{o}}+$ agonist + BDNF, $p=0.0280$, 30 $0.7 \mathrm{~mm}\left[\mathrm{Ca}^{2+}\right]_{\mathrm{o}}+$ agonist + BDNF $p=$ $0.0001,60^{\prime} 0.7 \mathrm{mM}\left[\mathrm{Ca}^{2+}\right]_{\mathrm{o}}+$ agonist $+\mathrm{BDNF} p=0.0072$, post hoc Dunnett; Fig. 10E,F). These results suggest that neurite growth is regulated through the GSK3-Tau pathway following activation of CaSR.

\section{Discussion}

Neurons use different signaling systems to diversify the read-out to a limited number of guidance cues, increasing possible growth and guidance responses. However, despite recent progress, our understanding of how signal integration induces growth and 


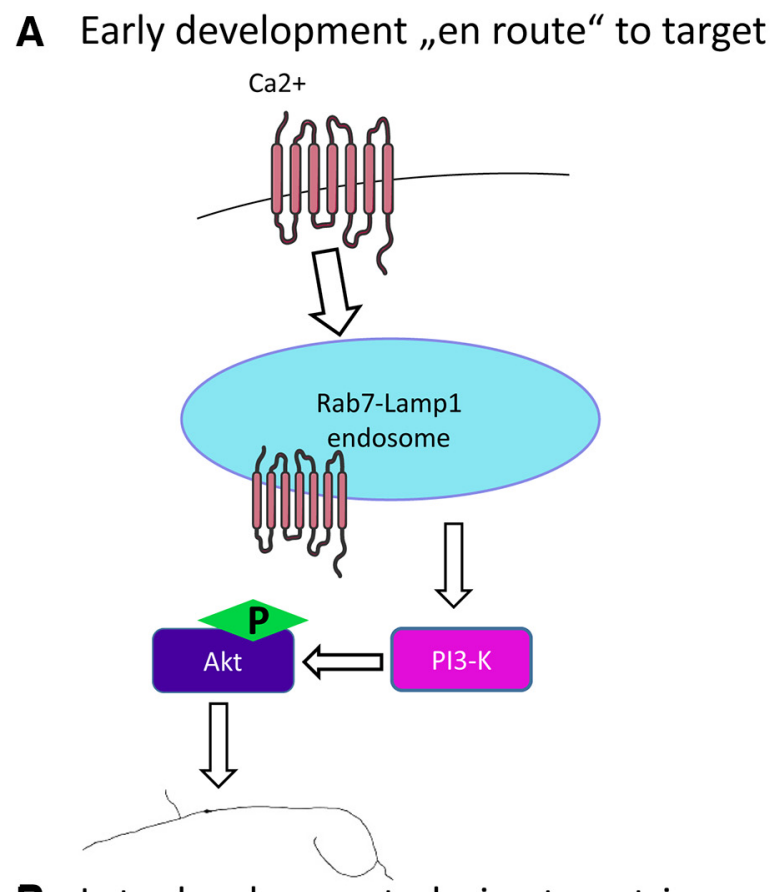

B Late development, during target innervation

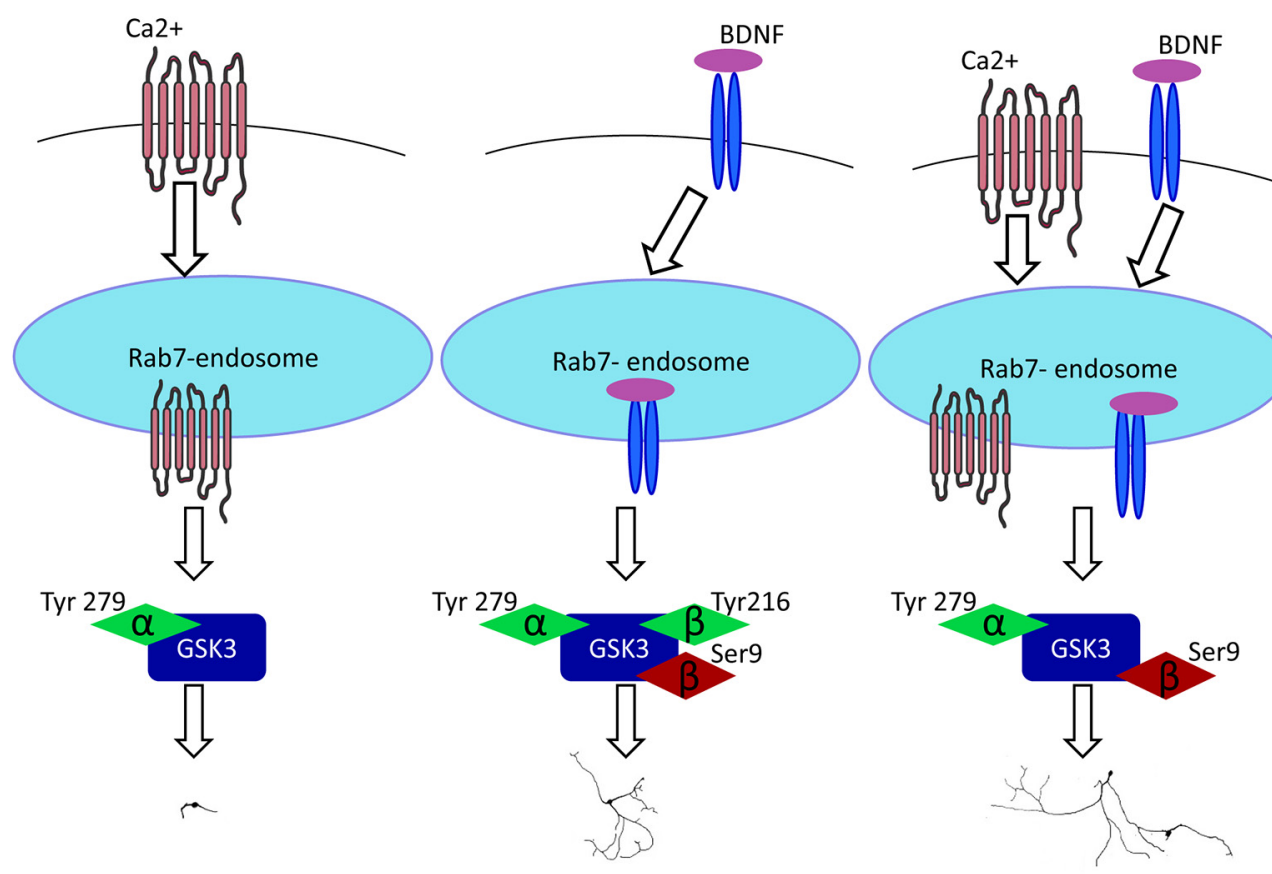

Figure 11. Summary of CaSR regulating growth in early nodose neurons and synergistic effects of CaSR/TrkB in late nodose neurons. $A$, Nodose neurons, which do not express TrkB, regulate growth by routing CaSR into Rab7 endosomes and activation of p-Akt. B, During target innervation, activation of CaSR leads to routing into Rab7 endosomes and phosphorylation of GSK3 $\alpha$ Tyr279. This phosphorylation only leads to minimal growth. Activation of TrkB leads to phosphorylation of GSK3 $\alpha$ Tyr279 and GSK3 $\beta$ Tyr216, as well as GSK3 $\beta$ Ser9. This interaction leads to an increase in growth compared with activation of CaSR alone. Activation of both CaSR and TrkB simultaneously leads to nonadditive effects by phosphorylating GSK3 $\alpha$ Tyr 279 and GSK3 $\beta$ Ser9. This coactivation significantly increases neurite growth compared with BDNF stimulation alone. Illustration of the CaSR was taken from Servier Medical Art templates, which are licensed under a Creative Commons Attribution 3.0 Unported License; https://smart.servier.com.

guidance remains incomplete. In this study, we show that CaSR regulates neural growth in two different ways: in isolation during early chick development and as a coreceptor of TrkB at later developmental stages. Coactivation of CaSR and TrkB leads to a change in downstream signaling of CaSR. During target innervation, CaSR and TrkB both localize to Rab7-late endosomes, coimmunoprecipitate, and phosphorylate GSK3 on specific residues, which causes GSK3 to cycle in its activity state. This change in activity subsequently results in cycling of the phosphorylation state of Tau, potentially modulating microtubule assembly and disassembly.

\section{Neurotrophic independence of cranial neurons}

The duration of neurotrophic independence for survival and the expression of TrkB in early cranial neurons, which are en route to their targets, correlate with target distance and with the arrival of 
axons in the vicinity of their targets (Vogel and Davies, 1991; Robinson et al., 1996).

In Figure 3C, we demonstrate that neurons become dependent on neurotrophic support for survival around Stage 24 (see also Robinson et al., 1996) and that the survival rate in absence of BDNF at Stage 30 (representing E6.5 to E7) is 35\%. Importantly, our results are in line with the observations of Vogel and Davies (1991) where the authors cultured different cranial sensory neurons in the absence of BDNF for up to $144 \mathrm{~h}$ and found a survival rate of $\sim 40 \%$.

However, why $\sim 30 \%-40 \%$ of neurons survive in the absence of BDNF remains unexplained. For survival experiments, neurons were cultured in low density, and we expected neurotrophins produced by neurons themselves to be diluted in the culture medium and therefore noneffective. However, supplementary data of Vizard et al. (2008) show that cultured SCG neurons show $\sim 20 \%$ survival in the absence of NGF and $\sim 40 \%$ survival in the presence of only $0.1 \mathrm{ng} / \mathrm{ml} \mathrm{NGF}$. Therefore, it is possible that some neurons respond to very low amounts of neurotrophic factors produced by neurons themselves.

Further, nodose neurons have been found to consist of several types of neurons, which show differences in their conductance velocity and their morphological appearance (Li and Schild, 2007; Lu et al., 2013). Apart from prosurvival signaling mediated by neurotrophins, an increase in spontaneous activity is commonly associated with a decreased rate of apoptosis (Golbs et al., 2011; Murase et al., 2011; Blanquie et al., 2017). For example, adult-generated neurons from the olfactory bulb have been shown to require intrinsic electrical properties to survive before and during their integration with their synaptic targets (Lin et al., 2010).

Thus, one possible mechanism to explain higher survival rates in nodose neurons could be that these (or a subset of these) neurons show intrinsic electrical activity for a certain amount of time to increase survival before and during target innervation.

\section{Signaling of CaSR during development}

Unfortunately, not much is known about $\left[\mathrm{Ca}^{2+}\right]_{\mathrm{o}}$ in the chicken embryo. One paper reported that calcium levels in the chicken blood serum (in milligrams $\mathrm{Ca} / 100 \mathrm{mg}$ ) rise during development (Narbaitz et al., 1973). In the rat, $\left[\mathrm{Ca}^{2+}\right]_{0}$ levels of cerebrospinal fluid declines from about $1.6 \mathrm{mM}$ in the fetus to $1.1 \mathrm{mM}$ in adults (Jones and Keep, 1988), showing that these embryos are hypercalcemic during development. Further, it is known that in humans, the fetus is hypercalcemic in their blood calcium levels compared to the mother (Kovacs and Kronenberg, 1997). Therefore, our findings suggest that the $\left[\mathrm{Ca}^{2+}\right]_{\mathrm{o}}$, to which nodose neurons are exposed to during development, constitutively stimulate neurite and axonal growth via CaSR.

To understand how signal integration of CaSR and TrkB functions, we took advantage of the fact that nodose neurons do not express TrkB until Hamburger-Hamilton Stage 23/24. We first investigated signaling of CaSR in the absence of TrkB during early development. In the absence of TrkB at Stage 22 (Davies et al., 1986; Davies, 1989; Vogel and Davies, 1991; Robinson et al., 1996), we show that activation of CaSR regulates neurite growth in vitro and in vivo.

Because trafficking and signaling are tightly linked (Dobrowolski and De Robertis, 2011; Pálfy et al., 2012; Harrington and Ginty, 2013), we evaluated the signaling cascade of CaSR as well as its localization to endosomes. Activation of CaSR leads to localization to late endosomes and phosphorylation of Akt but does not activate GSK3 or MAPK signaling, which is interesting since
CaSR was reported to activate the Mek-Erk pathway in various cell types (Holstein et al., 2004; Reyes-Ibarra et al., 2007; Vizard et al., 2015; Mizumachi et al., 2017). However, later in development, we observed that blocking Mek with PD98059 caused neurons to grow larger compared with $2.3 \mathrm{~mm}$ calcium, suggesting a developmental as well as a cell-specific response.

At the time of target innervation, nodose neurons become dependent on BDNF-TrkB signaling for growth and survival (Davies and Lindsay, 1985; Lindsay et al., 1985; Vogel and Davies, 1991; Robinson et al., 1996). At Stage 30, CaSR is unable to regulate growth when activated in isolation but enhances TrkB/ BDNF-mediated neurite growth. As mentioned, Trk receptors traffic in and signal from Rab7-positive endosomes (Kuruvilla et al., 2004; Deinhardt et al., 2006; Burk et al., 2017b). For CaSR to enhance TrkB-mediated growth, we hypothesized that both receptors need to be in close proximity to interact. The literature reports that CaSR is endocytosed and traffics through Rab11recycling endosomes, as well as being degraded through late endosomes (Holstein et al., 2004; Reyes-Ibarra et al., 2007; Zhuang et al., 2012; Ray, 2015). Indeed, in Stage 30 nodose neurons, CaSR colocalizes in late Rab7-positive endosomes and coimmunoprecipitates to and cotraffics with TrkB. Further, at Stage 30, CaSR switches its downstream signaling from Akt to GSK3 $\alpha$ Tyr279, a finding we are the first to report (discussed below).

How neurons switch their downstream signaling cascades from one protein to another is still debated. Because many proteins localize to endosomal membranes and can be targeted by multiple interconnected upstream players, mechanisms must exist to "insulate" the multitude of signals received by the cell. In the example of GSK3, insulation of signaling pathways has been proposed by regulation of the kinase that is used to prime a target for GSK3-mediated phosphorylation as well as other factors, such as the relative affinity of GSK3 for various target proteins (Dobrowolski and De Robertis, 2011). Our results suggest that the switch in CaSR signaling is spatiotemporally controlled, increasing the response range to different target cues over time. Still, which players regulate response mechanisms upstream of GSK3 or Akt, or how CaSR interacts with TrkB, direct or indirect, remains unanswered and will be subject of future research.

\section{Neurite growth regulation by GSK3 via signal integration of CaSR and TrkB}

Different growth and guidance cues use the Akt-GSK3 $\beta$ Ser9 pathway to regulate neurite growth (Yoshimura et al., 2005; Zhou et al., 2008; Bellon et al., 2010). However, we report, for the first time, that phosphorylation on GSK3 $\alpha$ Tyr279 and GSK3 $\beta$ Tyr216 is also involved in regulating neurite growth, which is interesting for two reasons. First, until now, the physiological significance of tyrosine phosphorylation on GSK3 $\alpha$ remained unclear, especially because this phosphorylation has been reported to be constitutive (Hughes et al., 1993). This, however, could be due to the cells generally being cultured in media containing physiological levels of calcium, ranging from 1.4 to 1.8 mM. Therefore, in any experimental setup using normal cell culture medium, CaSR would be fully active, thus explaining constitutive phosphorylation on GSK3 $\alpha$ Tyr279. Second, phosphorylation of GSK3 on its different residues changes the activity rates of GSK3 and therefore of its downstream targets. Phosphorylation of GSK3 on its tyrosine residues has been reported to increase activity of GSK3: Insulin treatment of SH-SY5Y cells caused increased GSK3 $\beta$ phosphorylation on Tyr216, increased GSK3 activity, and increased Tau phosphorylation (discussed below) (Hartigan and Johnson, 1999; Lesort et al., 1999). Therefore, 
BDNF-TrkB signaling phosphorylating GSK3 $\alpha$ Tyr279 as well as GSK $3 \beta$ Tyr 216 increases the activity of GSK3. Contrarily, BDNFinduced activation of PI3-kinase/Akt results in phosphorylation of Ser9 of GSK3 $\beta$, which inhibits GSK3 activity leading to the dephosphorylation of substrates (Cross et al., 1995; Cohen et al., 1997; Yoshimura et al., 2005). In our study, we found that the Akt-GSK $3 \beta$ Ser9 pathway also occurs when neurons were stimulated with BDNF, which reduces the activity of GSK3.

Therefore, our results report different "activity-increasing" and "activity decreasing" signals, and we found that the signal integration of TrkB and CaSR on GSK3 is not a sum of all incoming signals but a synergistic effect (for summary, see Fig. 11B).

Data from another study suggest that the interaction of CaSR/ Trk is a more general mechanism regulating neurite growth. Vizard et al. (2015) demonstrated that mouse SCG neurons cultured in the presence of $0.7 \mathrm{~mm}$ calcium containing $10 \mathrm{ng} / \mathrm{ml}$ of NGF (therefore, only activating TrkA) show low levels of p-Erk in immunostainings. Similarly, SCG neurons cultured in $2.3 \mathrm{~mm}$ calcium in the absence of NGF (therefore, only activating CaSR) also showed low levels of p-Erk. However, SCG neurons cultured in $2.3 \mathrm{~mm}$ calcium together with $10 \mathrm{ng} / \mathrm{ml}$ of NGF (therefore, TrkA and CaSR being activated simultaneously), caused a significant increase in p-Erk staining, suggesting that CaSR/Trk interaction and signaling also occur in other neurons. However, depending on neuronal subtype, downstream targets of these two receptors change: In nodose neurons, the main downstream target is GSK3, whereas in SCG neurons the Mek/Erk pathway is activated. Further, in organotypic slice cultures of mouse hippocampal neurons, transfection of CaSR-DN decreases branching of pyramidal neurons (Vizard et al., 2008). Because other studies show that the number, complexity, and length of primary dendrites in hippocampal neurons are dependent on BDNF (Ji et al., 2005; Burk et al., 2018), these findings suggest that branching of hippocampal pyramidal neurons could be facilitated by an interaction of CaSR and TrkB. Because CaSR/Trk interactions seem to be a general mechanism, we also tried to show interactions of CaSR and TrkB in mouse hippocampal neurons by overexpressing GFP-CaSR and RFP-TrkB. However, due to low transfection rates of CaSR and TrkB constructs, we did not find interaction. Performing coimmunoprecipitation from endogenous proteins was not possible because the CaSR-antibody does not work in Western blots.

\section{Tau activity regulating neurite growth}

So far, our results suggest that CaSR and TrkB regulate growth through activation and inactivation of GSK3, cycling it between activity states. This would suggest that downstream substrates, such as Tau, show cycling behavior in their phosphorylation state, which can be used as a read-out for microtubule assembly and disassembly, a key mechanism for neurite growth (Lindwall and Cole, 1984; Drechsel et al., 1992).

To link GSK3 activity to microtubule assembly, we tested whether Tau is activated downstream of CaSR when signaling in isolation as well as a coreceptor to TrkB. We found that Tau is phosphorylated downstream of CaSR and GSK3 activation and inactive in the presence of the CaSR and GSK3 antagonist Calhex 231 and Bio, respectively. In line with our hypothesis, we found that, when CaSR and TrkB are coactivated, Tau phosphorylation shows a cycling behavior, suggesting that CaSR and TrkB regulate GSK3 activity and inactivity. Thus, these results identify a novel regulatory pathway mediating neurite growth.

In conclusion, a deeper understanding of signal diversification in neurons will shed more light on neural circuit assembly and might help to develop tools for modulating neural regeneration. In addition, the effect of CaSR on neurite growth and target innervation together with TrkB may help to explain neurological and developmental deficits of patients with mutations of CaSR (Cole et al., 1990).

\section{References}

Bai M, Quinn S, Trivedi S, Kifor O, Pearce SH, Pollak MR, Krapcho K, Hebert SC, Brown EM (1996) Expression and characterization of inactivating and activating mutations in the human $\mathrm{Ca}^{2+}(\mathrm{o})$-sensing receptor. J Biol Chem 271:19537-19545.

Barford K, Deppmann C, Winckler B (2017) The neurotrophin receptor signaling endosome: where trafficking meets signaling. Dev Neurobiol 77:405-418.

Bashaw GJ, Klein R (2010) Signaling from axon guidance receptors. Cold Spring Harb Perspect Biol 2:a001941.

Bellon A, Luchino J, Haigh K, Rougon G, Haigh J, Chauvet S, Mann F (2010) VEGFR2 (KDR/Flk1) signaling mediates axon growth in response to semaphorin 3E in the developing brain. Neuron 66:205-219.

Blanquie O, Yang JW, Kilb W, Sharopov S, Sinning A, Luhmann HJ (2017) Electrical activity controls area-specific expression of neuronal apoptosis in the mouse developing cerebral cortex. Elife 6:e27696.

Bourne HR, Nicoll R (1993) Molecular machines integrate coincident synaptic signals. Cell 72 [Suppl]:65-75.

Brennan SC, Thiem U, Roth S, Aggarwal A, Fetahu IS, Tennakoon S, Gomes AR, Brandi ML, Bruggeman F, Mentaverri R, Riccardi D, Kallay E (2013) Calcium sensing receptor signalling in physiology and cancer. Biochim Biophys Acta 1833:1732-1744.

Burk K, Mire E, Bellon A, Hocine M, Guillot J, Moraes F, Yoshida Y, Simons M, Chauvet S, Mann F (2017a) Post-endocytic sorting of Plexin-D1 controls signal transduction and development of axonal and vascular circuits. Nat Commun 8:14508.

Burk K, Murdoch JD, Freytag S, Koenig M, Bharat V, Markworth R, Burkhardt S, Fischer A, Dean C (2017b) EndophilinAs regulate endosomal sorting of BDNF-TrkB to mediate survival signaling in hippocampal neurons. Sci Rep 7:2149.

Burk K, Ramachandran B, Ahmed S, Hurtado-Zavala JI, Awasthi A, Benito E, Faram R, Ahmad H, Swaminathan A, McIlhinney J, Fischer A, Perestenko P, Dean C (2018) Regulation of dendritic spine morphology in hippocampal neurons by copine-6. Cereb Cortex 28:1087-1104.

Chao MV (2003) Neurotrophins and their receptors: a convergence point for many signalling pathways. Nat Rev Neurosci 4:299-309.

Chauvet S, Cohen S, Yoshida Y, Fekrane L, Livet J, Gayet O, Segu L, Buhot MC, Jessell TM, Henderson CE, Mann F (2007) Gating of Sema3E/PlexinD1 signaling by neuropilin-1 switches axonal repulsion to attraction during brain development. Neuron 56:807-822.

Cohen P, Alessi DR, Cross DA (1997) PDK1, one of the missing links in insulin signal transduction? FEBS Lett 410:3-10.

Cole D, Forsythe CR, Dooley JM, Grantmyre EB, Salisbury SR (1990) Primary neonatal hyperparathyroidism: a devastating neurodevelopmental disorder if left untreated. J Craniofacial Genet Dev Biol 10:205-214.

Cornell RA, Kimelman D (1994) Combinatorial signaling in development. BioEssays 16:577-581.

Cross DA, Alessi DR, Cohen P, Andjelkovich M, Hemmings BA (1995) Inhibition of glycogen synthase kinase- 3 by insulin mediated by protein kinase B. Nature 378:785-789.

Davies AM (1989) Intrinsic differences in the growth rate of early nerve fibres related to target distance. Nature 337:553-555.

Davies AM, Lindsay RM (1985) The cranial sensory ganglia in culture: differences in the response of placode-derived and neural crest-derived neurons to nerve growth factor. Dev Biol 111:62-72.

Davies AM, Thoenen H, Barde YA (1986) The response of chick sensory neurons to brain-derived neurotrophic factor. J Neurosci 6:1897-1904.

Deinhardt K, Salinas S, Verastegui C, Watson R, Worth D, Hanrahan S, Bucci C, Schiavo G (2006) Rab5 and Rab7 control endocytic sorting along the axonal retrograde transport pathway. Neuron 52:293-305.

Dickson BJ (2002) Molecular mechanisms of axon guidance. Science 298: 1959-1964.

Dickson BJ, Gilestro GF (2006) Regulation of commissural axon pathfinding by slit and its Robo receptors. Annu Rev Cell Dev Biol 22:651-675.

Dobrowolski R, De Robertis EM (2011) Endocytic control of growth factor 
signalling: multivesicular bodies as signalling organelles. Nat Rev Mol Cell Biol 13:53-60.

Drechsel DN, Hyman AA, Cobb MH, Kirschner MW (1992) Modulation of the dynamic instability of tubulin assembly by the microtubule-associated protein Tau. Mol Biol Cell 3:1141-1154.

Dudanova I, Klein R (2013) Integration of guidance cues: Parallel signaling and crosstalk. Trends Neurosci 36:295-304.

Filbin MT (2006) Recapitulate development to promote axonal regeneration: good or bad approach? Philos Trans R Soc Lond B Biol Sci 361:1565-1574.

Gavaldà N, Gutierrez H, Davies AM (2009) Developmental switch in NF- B signalling required for neurite growth. Development 136:3405-3412.

Golbs A, Nimmervoll B, Sun JJ, Sava IE, Luhmann HJ (2011) Control of programmed cell death by distinct electrical activity patterns. Cereb Cortex 21:1192-1202.

Hamburger V, Hamilton HL (1992) A series of normal stages in the development of the chick embryo. Dev Dyn 195:231-272.

Harrington AW, Ginty DD (2013) Long-distance retrograde neurotrophic factor signalling in neurons. Nat Rev Neurosci 14:177-187.

Hartigan JA, Johnson GV (1999) Transient increases in intracellular calcium result in prolonged site-selective increases in Tau phosphorylation through a glycogen synthase kinase 3beta-dependent pathway. J Biol Chem 274:21395-21401.

Holstein DM, Berg KA, Leeb-Lundberg LM, Olson MS, Saunders C (2004) Calcium-sensing receptor-mediated ERK1/2 activation requires $\mathrm{G} \alpha$ i2 coupling and dynamin-independent receptor internalization. J Biol Chem 279:10060-10069.

Hughes K, Nikolakaki E, Plyte SE, Totty NF, Woodgett JR (1993) Modulation of the glycogen synthase kinase-3 family by tyrosine phosphorylation. ЕMBO J 12:803-808.

Ji Y, Pang PT, Feng L, Lu B (2005) Cyclic AMP controls BDNF-induced TrkB phosphorylation and dendritic spine formation in mature hippocampal neurons. Nat Neurosci 8:164-172.

Jones BL, Smith SM (2016) Calcium-sensing receptor: a key target for extracellular calcium signaling in neurons. Front Physiol 7:116.

Jones HC, Keep RF (1988) Brain fluid calcium concentration and response to acute hypercalcaemia during development in the rat. J Physiol 402:579-593.

Kovacs CS, Kronenberg HM (1997) Maternal-fetal calcium and bone metabolism during pregnancy, puerperium, and lactation. Endocr Rev 18:832-872.

Kuruvilla R, Zweifel LS, Glebova NO, Lonze BE, Valdez G, Ye H, Ginty DD (2004) A neurotrophin signaling cascade coordinates sympathetic neuron development through differential control of TrkA trafficking and retrograde signaling. Cell 118:243-255.

Lesort M, Jope RS, Johnson GV (1999) Insulin transiently increases Tau phosphorylation: involvement of glycogen synthase kinase- $3 \beta$ and fyn tyrosine kinase. J Neurochem 72:576-584.

Li BY, Schild JH (2007) Electrophysiological and pharmacological validation of vagal afferent fiber type of neurons enzymatically isolated from rat nodose ganglia. J Neurosci Methods 164:75-85.

Lin CW, Sim S, Ainsworth A, Okada M, Kelsch W, Lois C (2010) Genetically increased cell-intrinsic excitability enhances neuronal integration into adult brain circuits. Neuron 65:32-39.

Lindsay RM, Thoenen H, Barde YA (1985) Placode and neural crest-derived sensory neurons are responsive at early developmental stages to brainderived neurotrophic factor. Dev Biol 112:319-328.

Lindwall G, Cole RD (1984) Phosphorylation affects the ability of Tau protein to promote microtubule assembly. J Biol Chem 259:5301-5305.

Lu W, Yamamoto V, Ortega B, Baltimore D (2004) Mammalian Ryk is a Wnt coreceptor required for stimulation of neurite outgrowth. Cell 119:97-108.

Lu XL, Xu WX, Yan ZY, Qian Z, Xu B, Liu Y, Han LM, Gao RC, Li JN, Yuan M, Zhao CB, Qiao GF, Li BY (2013) Subtype identification in acutely dissociated rat nodose ganglion neurons based on morphologic parameters. Int J Biol Sci 9:716-727.

McClean MN, Mody A, Broach JR, Ramanathan S (2007) Cross-talk and decision making in MAP kinase pathways. Nat Genet 39:409-414.

Mire E, Mezzera C, Leyva-DíazE, Paternain AV, Squarzoni P, Bluy L, CastilloPaterna M, López MJ, Peregrín S, Tessier-Lavigne M, Garel S, Galcerán J,
Lerma J, López-Bendito G (2012) Spontaneous activity regulates Robo1 transcription to mediate a switch in thalamocortical axon growth. Nat Neurosci 15:1134-1143.

Mire E, Hocine M, Bazellières E, Jungas T, Davy A, Chauvet S, Mann F (2018) Developmental upregulation of Ephrin-B1 silences Sema3C/Neuropilin-1 signaling during post-crossing navigation of corpus callosum axons. Curr Biol 28:1768-1782.e4.

Mizumachi H, Yoshida S, Tomokiyo A, Hasegawa D, Hamano S, Yuda A, Sugii H, Serita S, Mitarai H, Koori K, Wada N, Maeda H (2017) Calcium-sensing receptor-ERK signaling promotes odontoblastic differentiation of human dental pulp cells. Bone 101:191-201.

Morales D, Kania A (2017) Cooperation and crosstalk in axon guidance cue integration: additivity, synergy, and fine-tuning in combinatorial signaling. Dev Neurobiol 77:891-904.

Murase S, Owens DF, McKay RD (2011) In the newborn hippocampus, neurotrophin-dependent survival requires spontaneous activity and integrin signaling. J Neurosci 31:7791-7800.

Pálfy M, Reményi A, Korcsmáros T (2012) Endosomal crosstalk: meeting points for signaling pathways. Trends Cell Biol 22:447-456.

Prehoda KE, Lim WA (2002) How signaling proteins integrate multiple inputs: a comparison of N-WASP and Cdk2. Curr Opin Cell Biol 14:149-154.

Narbaitz R, Bélanger LF, Hunt BJ (1973) Calcium regulation by the chick embryo: an experimental approach. Calcif Tissue Res 11:238-241.

Ray K (2015) Calcium-sensing receptor: trafficking, endocytosis, recycling, and importance of interacting proteins. In: Progress in molecular biology and translational science, pp 127-150. Amsterdam: Elsevier.

Reyes-Ibarra AP, García-Regalado A, Ramírez-Rangel I, Esparza-Silva AL, Valadez-Sánchez M, Vázquez-Prado J, Reyes-Cruz G (2007) Calciumsensing receptor endocytosis links extracellular calcium signaling to parathyroid hormone-related peptide secretion via a Rabl1a-dependent and AMSH-sensitive mechanism. Mol Endocrinol 21:1394-1407.

Robinson M, Adu J, Davies AM (1996) Timing and regulation of trkB and BDNF mRNA expression in placode-derived sensory neurons and their targets. Eur J Neurosci 8:2399-2406.

Ruat M, Traiffort E (2013) Roles of the calcium sensing receptor in the central nervous system. In: Best practice and research: clinical endocrinology and metabolism, pp 429-442. Amsterdam: Elsevier.

Saxena S, Bucci C, Weis J, Kruttgen A (2005) The small GTPase Rab7 controls the endosomal trafficking and neuritogenic signaling of the nerve growth factor receptor TrkA. J Neurosci 25:10930-10940.

Song M, Giza J, Proenca CC, Jing D, Elliott M, Dincheva I, Shmelkov SV, Kim J, Schreiner R, Huang SH, Castrén E, Prekeris R, Hempstead BL, Chao MV, Dictenberg JB, Rafii S, Chen ZY, Rodriguez-Boulan E, Lee FS (2015) Slitrk5 mediates BDNF-dependent TrkB receptor trafficking and signaling. Dev Cell 33:690-702.

Vizard TN, O’Keeffe GW, Gutierrez H, Kos CH, Riccardi D, Davies AM (2008) Regulation of axonal and dendritic growth by the extracellular calcium-sensing receptor. Nat Neurosci 11:285-291.

Vizard TN, Newton M, Howard L, Wyatt S, Davies AM (2015) ERK signaling mediates CaSR-promoted axon growth. Neurosci Lett 603:77-83.

Vogel KS, Davies AM (1991) The duration of neurotrophic factor independence in early sensory neurons is matched to the time course of target field innervation. Neuron 7:819-830.

Wen Z, Zheng JQ (2006) Directional guidance of nerve growth cones. Curr Opin Neurobiol 16:52-58.

Wu C, Lai CF, Mobley WC (2001) Nerve growth factor activates persistent Rap1 signaling in endosomes. J Neurosci 21:5406-5416.

Yoshimura T, Kawano Y, Arimura N, Kawabata S, Kikuchi A, Kaibuchi K (2005) GSK-3 $\beta$ regulates phosphorylation of CRMP- 2 and neuronal polarity. Cell 120:137-149.

Zhou B, Cai Q, Xie Y, Sheng ZH (2012) Snapin recruits dynein to BDNFTrkB signaling endosomes for retrograde axonal transport and is essential for dendrite growth of cortical neurons. Cell Rep 2:42-51.

Zhou Y, Gunput RA, Pasterkamp RJ (2008) Semaphorin signaling: progress made and promises ahead. Trends Biochem Sci 33:161-170.

Zhuang X, Northup JK, Ray K (2012) Large putative PEST-like sequence motif at the carboxyl tail of human calcium receptor directs lysosomal degradation and regulates cell surface receptor level. J Biol Chem 287: 4165-4176. 